\title{
Statistical properties of a sample of periodically variable B-type supergiants ${ }^{\star}$
}

\section{Evidence for opacity-driven gravity-mode oscillations}

\author{
K. Lefever ${ }^{1}$, J. Puls ${ }^{2}$, and C. Aerts ${ }^{1,3}$ \\ 1 Instituut voor Sterrenkunde, K.U. Leuven, Celestijnenlaan 200D, 3001 Leuven, Belgium \\ e-mail: karolien@ster.kuleuven.be \\ 2 Universitätssternwarte München, Scheinerstr. 1, 81679 München, Germany \\ 3 Departement Astrofysica, Radboud Universiteit van Nijmegen, PO Box 9010, 6500 GL Nijmegen, The Netherlands
}

Received 14 July 2006/ Accepted 3 November 2006

\begin{abstract}
Aims. We have studied a sample of 28 periodically variable B-type supergiants selected from the HIPPARCOS mission and 12 comparison stars covering the whole B-type spectral range. Our goal is to test if their variability is compatible with opacity-driven non-radial oscillations.

Methods. We have used the NLTE atmosphere code FASTWIND to derive the atmospheric and wind parameters of the complete sample through line profile fitting. We applied the method to selected $\mathrm{H}, \mathrm{He}$, and Si line profiles, measured with the high resolution CES spectrograph attached to the ESO CAT telescope in La Silla, Chile.

Results. From the location of the stars in the $\left(\log T_{\text {eff }}, \log g\right)$ diagram, we suggest that variability of our sample supergiants is indeed due to the gravity modes resulting from the opacity mechanism. We find nine of the comparison stars to be periodically variable as well, and suggest them to be new $\alpha$ Cyg variables. We find marginal evidence of a correlation between the amplitude of the photometric variability and the wind density. We investigate the wind momentum-luminosity relation for the whole range of B spectral type supergiants, and find that the later types (>B5) perfectly follow the relation for A supergiants. Additionally, we provide a new spectral type $-T_{\text {eff }}$ calibration for B supergiants.

Conclusions. Our results imply the possibility of probing internal structure models of massive stars of spectral type B through seismic tuning of gravity modes.
\end{abstract}

Key words. stars: atmospheres - stars: early-type - stars: fundamental parameters - stars: mass-loss - stars: oscillations stars: variables: general

\section{Introduction}

One of the remarkable by-products of the ESA HIPPARCOS mission was the discovery of a large amount of new periodically variable B stars, including almost a hundred new slowly pulsating B stars (SPBs hereafter) and 29 periodically variable B-type supergiants (Waelkens et al. 1998; Aerts et al. 1999; Mathias et al. 2001). The photometric behaviour of different kinds of evolved massive stars were analysed in detail from HIPPARCOS data. VanLeeuwen et al. (1998) performed an analysis of 24 known B- to G-type variable supergiants and found periods of tens of days for each of them, in agreement with previous ground-based studies. Marchenko et al. (1998), on the other hand, focused on the HIPPARCOS variability of 141 O-type and WR stars and noted the remarkable variety of variability, with very diverse causes, within that sample. The study of Waelkens et al. (1998) is quite different in this respect, as they came up with a sample of 29 new variable B supergiants exhibiting clear periodic microvariations at relatively short periods of one to a few days. The current paper contains a follow-up study of the latter sample. Our goal is to evaluate the suggestion by Waelkens et al. (1998) that these periodically variable B-type supergiants

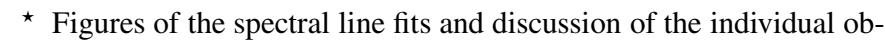
jects, Appendices A, B and Table 6 are only available in electronic form at http://www . aanda.org experience oscillations excited by the opacity mechanism, in analogy to main sequence $B$ stars.

The suggestion of the occurrence of non-radial oscillation modes in massive supergiants is not new. Microvariations with amplitudes between a hundredth and a tenth of a magnitude in the visual, and periods ranging from some 5 to $100 \mathrm{~d}$, have been found in numerous supergiants of spectral type OBA, termed $\alpha$ Cyg variables (Sterken 1977, 1983; Burki et al. 1978; van Genderen et al. 1989; Lamers et al. 1998; van Genderen 2001). Burki (1978) considered a sample of 32 B- to G-type supergiants and derived an empirical semi-period-luminositycolour (PLC) relation (see his Eq. (5)), from which he suggested the variability to be caused by oscillations rather than mass loss. Lovy et al. (1984) indeed managed to come up with a theoretical PLC relation in agreement with the observed one for this type of stars. However, only $40 \%$ of the variable supergiants have periods compatible with the radial fundamental mode, while the majority must exhibit a different kind of oscillation mode. Kaufer et al. (1997) made an extensive high-resolution spectroscopic study of 6 BA-type supergiants, which they monitored for several years. They concluded that the variability patterns are extremely complex and point towards cyclical variations in the radial velocities. From CLEANing the periodograms of the radialvelocity curves, they derived multiple periods and assigned them to non-radial oscillations because the traveling features in the 
dynamical spectra turned out to be incompatible with the rotational periods of the stars.

Glatzel \& Kiriakidis (1993) interpreted the periodic variability of supergiants with masses above $40 M_{\odot}$ in terms of strangemode instabilities and showed the classical opacity mechanism to be unimportant in such objects. Glatzel et al. (1999) subsequently performed detailed calculations for stars with $M=$ $64 M_{\odot}$ and predicted irregular velocity and luminosity variations with time scales of 1 to $20 \mathrm{~d}$. They also proposed that such massive stars undergo pulsationally-driven mass loss. It is not clear how their result will change for stars in the mass range of 10 to $30 M_{\odot}$, which is the transition region from low to large massloss rates due to line driving. Therefore, the periodic variations of the B supergiants found by Waelkens et al. (1998) might still be due to the classical opacity mechanism, since the instability strips of the $\beta$ Cep stars and the SPBs were found to extend to higher luminosities shortly after the discovery paper (Pamyatnykh 1999; Balona \& Dziembowski 1999).

Waelkens et al. (1998) positioned the new periodic B supergiants in the HR diagram on the basis of multicolour photometric calibrations (accurate parallaxes are not available) and found them to be situated between the SPBs and previously known $\alpha$ Cyg-type variables (see their Fig. 2). Oscillations were not predicted in that part of the HR diagram. Given the uncertainty in the effective temperature and luminosity, Aerts (2000a) tried to improve upon the fundamental parameter determination by constructing the spectral energy distribution of the stars as a better diagnostic to derive their effective temperature and gravity. This did not improve the large uncertainties on the latter parameters, however. Nevertheless, the sample selected by Waelkens et al. (1998) remained the most valuable one to observationally investigate the occurrence of gravity modes in supergiant stars because it is unbiased in the sense that the stars were not at all selected to be observed with HIPPARCOS on the basis of variability. For this reason, we conducted an extensive spectroscopic campaign for the sample, with the goal of performing line-profile fitting as a way to estimate the fundamental parameters of the stars with unprecedented precision. We report upon the analysis of these new data, and the position of the stars with respect to the instability strip of gravity modes, in the current work.

The questions we will address here are the following. We elaborate further on the HIPPARCOS data to search for multiperiodic variability, which is a natural property of non-radial oscillators. From selected $\mathrm{H}, \mathrm{He}$, and Si line profiles, we derive the physical parameters (effective temperature, gravity, luminosity, mass loss, rotational velocity, etc.) of the stars in the sample by Waelkens et al. (1998) using high-quality spectroscopic data. From this, we derive their position in the HR diagram with high precision and check if the stars lie within the instability strips of gravity mode oscillations predicted by Pamyatnykh (1999) and Saio et al. (2006). During our analysis we also look for asymmetries in the metallic line profiles. Further, we search for correlations between the physical parameters and the photometric variability. In particular, we investigate if there is any connection between the observed peak-to-peak amplitude and frequency of the light variability, the wind density, and the rotation of the stars. Finally, we investigate the wind momentum-luminosity relation (WLR) for the complete B-type spectral range.

\section{Observations and data reduction}

We selected all southern stars of luminosity class I or II brighter than 9th mag in the sample by Waelkens et al. (1998), which fitted the observation window of the assigned telescope time. This concerns 21 stars. To this we added 10 more candidate $\alpha$ Cyg variables from the Catalogue of Periodic Variables of HIPPARCOS (ESA 1997), in such way that the complete sample fully covers the B-type spectral range. Waelkens et al. (1998) were unable to assign them to one of the five considered classes ( $\beta$ Cep stars, SPBs, chemically peculiar stars, B-type supergiants, Be stars), see also Kestens (1998). Our period analysis and their spectral type clearly point towards $\alpha$ Cyg variables, however.

These 31 targets (for spectral types, see Table 6 and discussion in Sect. 7) were added to the long-term spectroscopic monitoring programme of periodic B stars conducted at Leuven University (Aerts et al. 1999). The spectra of the stars were gathered with the CES spectrograph attached to the CAT telescope at La Silla during numerous observing runs spread over two years. For most targets, we obtained two exposures of the $\mathrm{H} \alpha$ line in different seasons (to check for its variability), one of the $\mathrm{H} \gamma$ line, one of the He I $6678 \AA$ line, and one of the He I4471 $\AA$ line. Besides these, we observed one silicon line for each star with the goal of obtaining an accurate temperature estimate. Depending on spectral type, this is either the Si II $4130 \AA$ doublet (late B-type) or the Si III $4560 \AA$ triplet (early B-type).

The spectra were reduced in the standard way, i.e., by means of flatfielding, wavelength calibration through $\mathrm{Th}-\mathrm{Ar}$ exposures, and rectification of the continuum through a cubic spline fit. The resolution amounts to 70000 and the exposure times range from 3 to $50 \mathrm{~min}$, resulting in a high signal-to-noise ratio of about 250. It became immediately evident that the three stars HD 71913, HD 157485, and HD 165812 were misclassified in the Bright Star Catalogue (BSC) as supergiants. They turned out to be new $\beta$ Cep stars. These have been studied in detail by Aerts (2000b) and are not included here. This finally leads to 28 sample supergiant stars.

To assess the importance of having periodic light variability in our sample, we have additionally selected 12 bright B supergiants from the BSC, again chosen to cover the complete B-type spectral range. These variables were not classified as periodic variable by the HIPPARCOS classification algorithm. While for some of these bright objects stellar parameters are available in the literature, we have invested in collecting their spectra as well, to treat these stars in the same way as the sample stars.

\section{Photometric variability}

The HIPPARCOS data of the 40 targets were subjected to detailed period search by means of the Scargle (1982) and Phase Dispersion Minimisation (Stellingwerf 1978) methods. In Fig. 1, we show the phase diagrams for the dominant frequency for two representative cases. The detailed results of the frequency analyses are provided in Tables 1 and 2. Note that $P_{2}$ is derived from the residuals, after prewhitening with $P_{1}$ and that the total variance reduction is obtained from a harmonic fit to the data with both periods. The differences between the largest and the smallest observed magnitude are indicated by the observed peak-topeak values $\Delta H_{\mathrm{P}, \mathrm{obs}}$.

For most targets we recovered the main period found by Waelkens et al. (1998), but not for all of them. For six stars, the first harmonic of the main frequency was also needed to obtain an accurate fit to the HIPPARCOS data (see, e.g., Fig. 1). We found evidence for multiperiodicity for eleven stars. The detected periods range from 1.15 to $25 \mathrm{~d}$, with only four stars having a period longer than $10 \mathrm{~d}$. Thus we confirm that most of the 
HD 96880
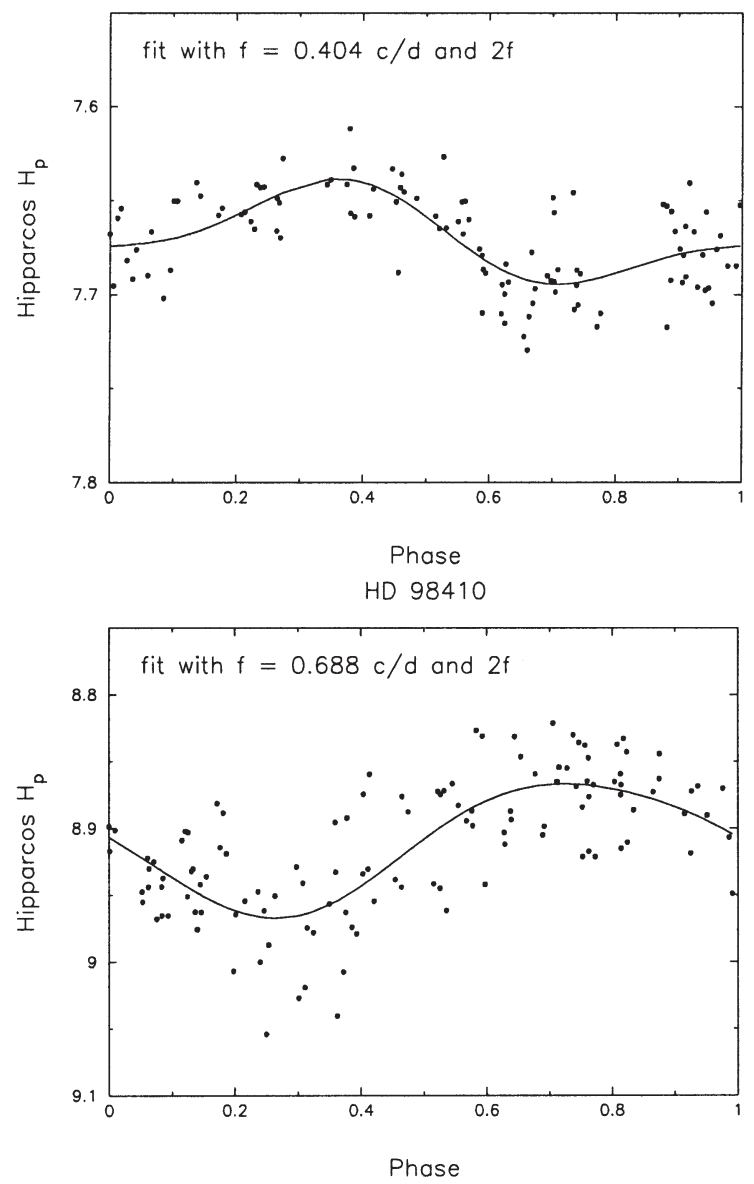

Fig. 1. The HIPPARCOS lightcurve folded according to the dominant frequency for the stars HD 96880 (B1 Ia) and HD 98410 (B2.5 Ib/II). The dots are the observations and the full line is a least-squares fit for the indicated frequency and its first harmonic.

28 sample stars have periods that are significantly shorter than the ones of other $\alpha$ Cyg variables. We do also find short periods (less than $10 \mathrm{~d}$ ) for some comparison stars, though they were not classified as periodic by the HIPPARCOS team. When comparing the observed periods and peak-to-peak amplitudes between the sample and comparison stars, seven out of twelve comparison stars have lower peak-to-peak variations, whereas the other five seem to have periods and amplitudes comparable to the ones detected in our target sample.

\section{Spectroscopic analysis: results from line profile fitting}

\section{1. $v$ sin $i$ from metallic lines}

The projected rotational velocity, $v \sin i$, was found from the automated tool developed by Simón-Díaz et al. (2006), which is based on a Fourier method to separate the effects of rotational broadening and macro-turbulence (which can be significant in B-type supergiants, cf. Ryans et al. 2002). This method was first described by Gray (1973) and reviewed in Gray (1978). It is discussed in detail more recently by Piters et al. (1996).

Weak metallic lines are the best diagnostic to derive $v \sin i$, since they are (by definition) free of saturation effects and least affected by collisional broadening. We have the following lines at our disposal: either Si II 4128-4130 ̊̊ or Si III 4552-4567$4574 \AA$ (depending on spectral type), Mg II $4481 \AA$ (in the same spectral order as He I $4471 \AA$ A) and, for slow rotators, also C II 6578-6582 $\AA$ near $\mathrm{H} \alpha$. Besides these primary diagnostics, other, even weaker metallic lines can also often be found.

Table 3 lists our results for $v \sin i$, together with its standard deviation and the number of lines we used. For the 12 comparison objects, not all selected orders were measured, and hence only a few lines could be used. For five stars we even lack useful metallic lines. Three out of these five (HD 64760, HD 157246, and HD 165024) have blended metal lines due to their fast rotation, and we adopt a mean value for $v \sin i$ as provided by SIMBAD. For the other two objects (HD 51110 and HD 147670), no value is given in SIMBAD, and it was not possible to use other lines to measure this quantity: for HD 51110 the He I lines were too weak and peculiar, whereas for HD 147670 only $\mathrm{H} \alpha$ had been secured.

The occurrence of asymmetries in the line profiles may reveal the presence of time-dependent pulsational broadening (see, e.g., Aerts \& De Cat 2003). In the current sample, clear asymmetries in the Si lines were detected only for HD 54764, HD 92964, and HD 109867. Most probably, they are related to binarity (HD 54764) or to a large mass-loss rate (HD 92964), which in both cases affect the photospheric lines in an asymmetric way. Only for HD 109867, we can speculate about a relation between line asymmetry and pulsational variability.

\subsection{Determination of physical parameters}

To investigate the position of our sample stars in the HR diagram on a solid basis, we determine the fundamental parameters of the stars from our high-resolution spectroscopic follow-up data. For this purpose, we use the non-LTE, spherically symmetric model atmosphere code FASTWIND (Fast Analysis of STellar atmospheres with WINDs), which enables us to derive atmospheric and wind parameters. The code was first introduced by Santolaya-Rey et al. (1997) and has meanwhile been updated for a number of improvements. The most important ones concern the inclusion of line blocking/blanketing effects and the calculation of a consistent temperature structure by exploiting the condition of flux-conservation in the inner and the electron thermal balance (e.g., Kubát et al. 1999) in the outer part of the atmosphere. A detailed description of the code was given by Puls et al. (2005), where representative results have also been compared with those from alternative NLTE codes, CMFGEN (Hillier \& Miller 1998), and WM-basic (Pauldrach et al. 2001). Meanwhile, a number of spectroscopic investigations of earlytype stars were performed by means of FASTWIND, both in the optical (e.g., Herrero et al. 2002; Trundle et al. 2004; Repolust et al. 2004; Massey et al. 2004, 2005; Mokiem et al. 2005, 2006) and in the NIR (Repolust et al. 2005).

As stated earlier, for most stars two hydrogen lines $(\mathrm{H} \alpha$ and $\mathrm{H} \gamma$ ), two $\mathrm{He} \mathrm{I}$ lines (the triplet line $4471 \AA$ and the singlet line $6678 \AA$ ), and one silicon multiplet (Si III 4552-4567-4574 for the early types, up to B2, and Si II 4128-4130 for the later spectral types) have been observed. The choice to measure these specific lines has not been made randomly, but is based on their well-known specific dependency on one or more of the basic parameters we want to unravel. The model atom for silicon used in this investigation is the same as used and described by Trundle et al. (2004) in their analysis of SMC B supergiants.

In Figs. 2-4, we show the isocontour levels of equivalent line width of Si II 4128, Si III 4552, and He I 4471, 
Table 1. Results of the period analyses for the 28 sample stars. Periods $P_{i}$ are expressed in $\mathrm{d}$ and have an accuracy better than $0.001 \mathrm{~d}$. The amplitudes $A_{i}$ and their $1 \sigma$ error, $\sigma_{A_{i}}$, are given in mmag. When the frequency's first harmonic is present in the lightcurve, the label "yes" occurs in Col. " $H$ ". Column "v.r." indicates the variance reduction of the harmonic fit, in \%. Observed peak-to-peak values are given in mmag.

\begin{tabular}{rrrcccccccc}
\hline \hline HD & $P_{1}$ & $A_{1} \pm \sigma_{A_{1}}$ & $H$ & v.r. & $P_{2}$ & $A_{2} \pm \sigma_{A_{2}}$ & $H$ & v.r. & (Total v.r.) & $\Delta H_{\text {P,obs }}$ \\
\hline 47240 & 1.730 & $29 \pm 4$ & no & $60 \%$ & & & & & & 10 \\
51110 & 2.315 & $61 \pm 6$ & no & $53 \%$ & & & & & & 150 \\
53138 & 24.390 & $45 \pm 5$ & no & $49 \%$ & 3.690 & $35 \pm 4$ & no & $45 \%$ & $(73 \%)$ & 100 \\
54764 & 2.695 & $17 \pm 4$ & no & $33 \%$ & & & & & & 50 \\
68161 & 16.949 & $25 \pm 2$ & no & $68 \%$ & & & & & & 50 \\
80558 & 1.695 & $38 \pm 6$ & no & $42 \%$ & 5.814 & $26 \pm 5$ & no & $25 \%$ & $(57 \%)$ & 90 \\
89767 & 1.153 & $28 \pm 4$ & no & $34 \%$ & & & & & & 100 \\
91024 & 2.398 & $36 \pm 6$ & no & $42 \%$ & & & & & & 100 \\
91943 & 6.452 & $24 \pm 4$ & no & $40 \%$ & & & & & & 70 \\
92964 & 14.706 & $43 \pm 5$ & no & $45 \%$ & 2.119 & $36 \pm 5$ & no & $43 \%$ & $(71 \%)$ & 110 \\
93619 & 4.310 & $27 \pm 6$ & no & $24 \%$ & & & & & & 100 \\
94367 & 7.937 & $48 \pm 5$ & yes & $58 \%$ & 4.329 & $27 \pm 5$ & no & $34 \%$ & $(73 \%)$ & 100 \\
94909 & 16.949 & $37 \pm 6$ & no & $36 \%$ & 1.256 & $25 \pm 4$ & no & $28 \%$ & $(54 \%)$ & 110 \\
96880 & 2.475 & $46 \pm 6$ & yes & $47 \%$ & & & & & & 120 \\
98410 & 1.453 & $97 \pm 8$ & yes & $53 \%$ & 8.696 & $40 \pm 8$ & yes & $45 \%$ & $(75 \%)$ & 230 \\
102997 & 2.688 & $29 \pm 6$ & yes & $33 \%$ & 2.976 & $23 \pm 4$ & yes & $37 \%$ & $(58 \%)$ & 110 \\
105056 & 2.899 & $41 \pm 8$ & no & $36 \%$ & 7.299 & $51 \pm 8$ & yes & $46 \%$ & $(67 \%)$ & 130 \\
106343 & 3.650 & $27 \pm 6$ & yes & $38 \%$ & 3.906 & $23 \pm 4$ & no & $31 \%$ & $(58 \%)$ & 110 \\
108659 & 5.076 & $22 \pm 4$ & no & $30 \%$ & & & & & & 80 \\
109867 & 4.484 & $32 \pm 3$ & no & $46 \%$ & 4.785 & $20 \pm 3$ & no & $30 \%$ & $(63 \%)$ & 80 \\
111990 & 2.890 & $30 \pm 4$ & no & $26 \%$ & & & & & & 120 \\
115363 & 3.077 & $38 \pm 8$ & no & $22 \%$ & & & & & & 120 \\
141318 & 1.466 & $16 \pm 2$ & no & $35 \%$ & & & & & & 50 \\
147670 & 5.435 & $66 \pm 4$ & no & $68 \%$ & & & & & & 110 \\
148688 & 6.329 & $46 \pm 6$ & no & $61 \%$ & 1.845 & $24 \pm 4$ & no & $42 \%$ & $(79 \%)$ & 90 \\
154043 & 2.874 & $30 \pm 7$ & no & $34 \%$ & & & & & & 80 \\
168183 & 2.105 & $60 \pm 5$ & no & $49 \%$ & & & & & & 150 \\
170938 & 5.618 & $94 \pm 18$ & yes & $61 \%$ & & & & & & 110 \\
\hline
\end{tabular}

Table 2. Results of the period analyses for the 12 comparison stars. Notations and units are the same as in Table 1.

\begin{tabular}{rrrccrrrrrr}
\hline \hline HD & $P_{1}$ & $A_{1} \pm \sigma_{A_{1}}$ & $H$ & v.r. & $P_{2}$ & $A_{2} \pm \sigma_{A_{2}}$ & $H$ & v.r. & (Total v.r.) & $\Delta H_{\mathrm{P}, \text { obs }}$ \\
\hline 46769 & 0.1122 & $9 \pm 2$ & no & $40 \%$ & & & & & & 22 \\
58350 & 6.6313 & $47 \pm 6$ & no & $39 \%$ & & & & & & 145 \\
64760 & 2.8090 & $11 \pm 2$ & no & $34 \%$ & 1.8447 & $9 \pm 2$ & no & $33 \%$ & $(56 \%)$ & 28 \\
75149 & 1.2151 & $33 \pm 5$ & no & $43 \%$ & 2.2143 & $20 \pm 4$ & no & $34 \%$ & $(63 \%)$ & 100 \\
86440 & 6.1996 & $9 \pm 2$ & yes & $51 \%$ & 0.2371 & $5 \pm 2$ & no & $22 \%$ & $(62 \%)$ & 36 \\
106068 & 4.2644 & $42 \pm 5$ & no & $46 \%$ & 25.1889 & $24 \pm 4$ & no & $31 \%$ & $(64 \%)$ & 110 \\
111904 & 3.3389 & $30 \pm 4$ & no & $38 \%$ & 19.1205 & $19 \pm 3$ & no & $32 \%$ & $(58 \%)$ & 110 \\
125288 & 8.0906 & $9 \pm 2$ & no & $21 \%$ & & & & & & 38 \\
149038 & 0.6390 & $19 \pm 3$ & no & $61 \%$ & & & & & & 37 \\
157038 & 3.6430 & $48 \pm 7$ & no & $68 \%$ & 1.5432 & $27 \pm 3$ & no & $50 \%$ & $(84 \%)$ & 100 \\
157246 & 1.1811 & $10 \pm 2$ & no & $40 \%$ & 0.1281 & $9 \pm 2$ & no & $40 \%$ & $(64 \%)$ & 20 \\
165024 & 2.7693 & $6 \pm 1$ & no & $28 \%$ & 0.8455 & $6 \pm 1$ & no & $24 \%$ & $(46 \%)$ & 24 \\
\hline
\end{tabular}

respectively, and their dependence on the effective temperature and surface gravity based on an extensive grid of synthetic models (see below). These figures show that Si II is a very good temperature indicator for B-type stars with an effective temperature below $20000 \mathrm{~K}$ (Fig. 2). From then on, Si III takes over as a temperature diagnostic (Fig. 3). Used in parallel, both silicon multiplets could be used to infer information about the silicon abundance. Since, however, we always have only one of the two at our disposal, we adopt a solar silicon abundance for our study $(\log (\mathrm{Si} / \mathrm{H})=-4.45$ by number, cf. Grevesse \& Sauval 1998, and references therein), which has changed only marginally (to $\log (\mathrm{Si} / \mathrm{H})=-4.49)$ in the recent update of the solar composition (Asplund et al. 2005).

The silicon abundance in B stars is heavily disputed. Depending on sample and method, values range from roughly solar (Gies \& Lambert 1992; Gummersbach et al. 1998;
Rolleston et al. 2000) to a depletion by typically 0.3 dex (Kilian 1992; Kilian et al. 1994; McErlean et al. 1999; Daflon \& Cunha 2004 ), in both cases with variations by \pm 0.2 dex. Analyses of individual objects by Urbaneja (2004) and Przybilla et al. (2006) indicate a rather large scatter, again in the same range of values. In view of this uncertainty, and the fact that Crowther et al. (2006, hereafter CR06) in his analysis of Galactic B supergiants found no obvious problems in using solar values, we also adopted this value. We will report on the influence of this assumption on the final outcome later on.

He I 4471 serves several purposes: for early B-types it is a good gravity indicator (with a certain sensitivity to temperature as well), whereas for the later B-types $\left(T_{\text {eff }}<15000 \mathrm{~K}\right)$ it becomes progressively independent of gravity, but can be used to constrain the temperatures perfectly (Fig. 4). In those cases where the effective temperatures (from $\mathrm{Si}$ ) and the gravity 
Table 3. Projected rotational velocity, $v \sin i$, and its standard deviation (in $\mathrm{km} \mathrm{s}^{-1}$ ) for all 40 sample stars, determined via the Fourier transform of metallic lines. When no metallic lines or only blended lines are available, the corresponding values from SIMBAD are given in italics. Column "n.o.l." gives the number of lines used to determine the mean value of $v \sin i$ and its standard deviation.

\begin{tabular}{rrrr|rrrr}
\hline \hline HD & $v \sin i$ & s.d. & n.o.l. & HD & $v \sin i$ & s.d. & n.o.l. \\
\hline 46769 & 68 & 5 & 2 & 102997 & 39 & 8 & 13 \\
47240 & 94 & 9 & 6 & 105056 & 61 & 21 & 10 \\
51110 & $n / a$ & & & 106068 & 26 & 5 & 6 \\
53138 & 38 & 4 & 14 & 106343 & 44 & 7 & 14 \\
54764 & 108 & 15 & 10 & 108659 & 29 & 5 & 12 \\
58350 & 37 & 5 & 4 & 109867 & 50 & 14 & 16 \\
64760 & 220 & & & 111904 & 32 & 9 & 4 \\
68161 & 17 & 2 & 20 & 111990 & 36 & 6 & 6 \\
75149 & 30 & 8 & 4 & 115363 & 55 & 12 & 8 \\
80558 & 28 & 5 & 17 & 125288 & 25 & 4 & 4 \\
86440 & 20 & 6 & 5 & 141318 & 32 & 3 & 8 \\
89767 & 47 & 6 & 12 & 147670 & $n / a$ & & \\
91024 & 25 & 6 & 23 & 148688 & 50 & 11 & 13 \\
91943 & 48 & 7 & 12 & 149038 & 57 & 4 & 2 \\
92964 & 31 & 6 & 17 & 154043 & 37 & 9 & 8 \\
93619 & 47 & 13 & 11 & 157038 & 41 & 5 & 4 \\
94367 & 31 & 4 & 15 & 157246 & 302 & & \\
94909 & 64 & 10 & 11 & 165024 & 95 & & \\
96880 & 44 & 9 & 14 & 168183 & 124 & 16 & 7 \\
98410 & 31 & 3 & 10 & 170938 & 51 & 6 & 10 \\
\hline
\end{tabular}

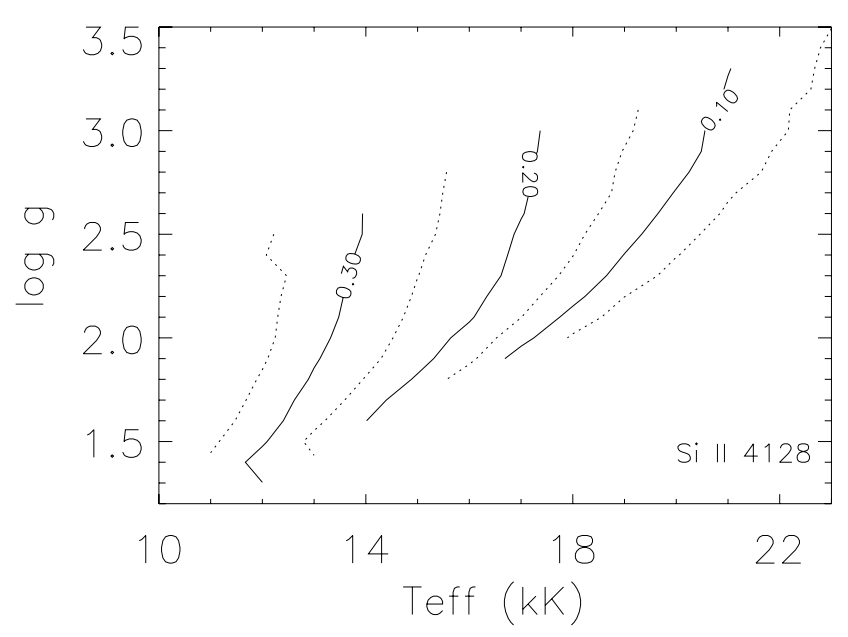

Fig. 2. Isocontour levels of equivalent line width (expressed in Angstrom) for Si II 4128 (solar Si abundance, negligible mass loss), demonstrating that this line is a good temperature indicator up to $20000 \mathrm{~K}$.

(from $\mathrm{H} \gamma$, see below) are well defined, both He I lines (He I 4471 and He I 6678) are useful to constrain the helium content, as well as to check for the overall consistency of the results, which in any case is the primary purpose of the second He I (singlet) line. The recent debate on the difficulty of using He I singlet lines as diagnostic tools (due to subtle line overlap effects of the He I resonance line in the FUV, Najarro et al. 2006) is (almost) irrelevant in the present context, since it concerns only spectral types of $\mathrm{B} 0$ and hotter.

Of course, He I 4471 is not our primary gravity indicator. As usual, we employ the Balmer line wings for this purpose, in our case, particularly the wings of $\mathrm{H} \gamma$. Since the $\mathrm{H} \alpha$ line is formed further out in the atmosphere, it is affected by the stellar wind, and, for larger wind densities, displays the typical emission or P Cygni type profile. Depending on the mass-loss rate,

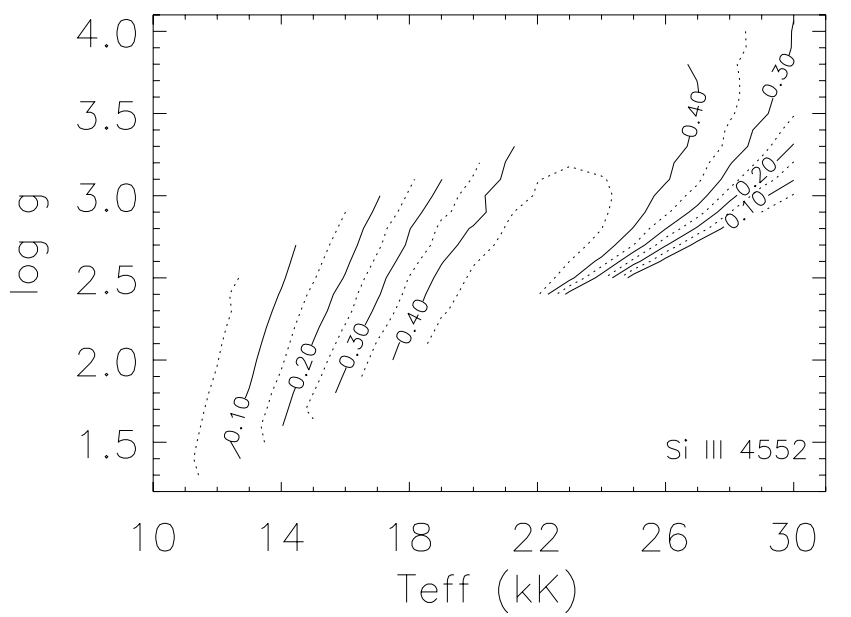

Fig. 3. As Fig. 2, but for Si III 4552. At temperatures around $23000 \mathrm{~K}$, $\mathrm{Si}$ III 4552 reaches a maximum in equivalent line width. The isocontours show that from this maximum, theoretical line profiles can behave similarly towards lower and higher temperatures, causing a dichotomy in the determination of the effective temperature, when we lack Si II or Si IV.

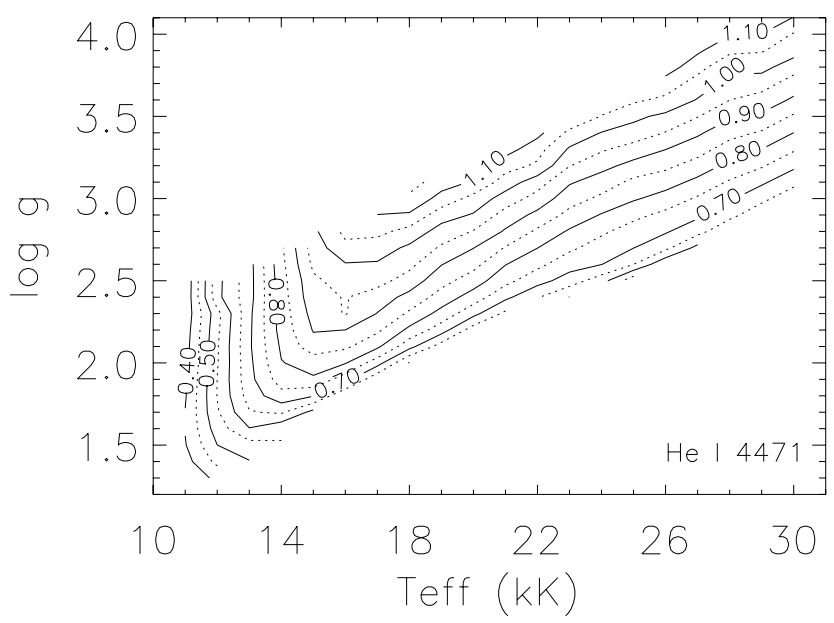

Fig. 4. Isocontour levels of equivalent line width (expressed in Angstrom) for He I $4471(n(\mathrm{He}) / n(\mathrm{H})=0.1$, negligible mass loss $)$. In the cool B-type regime, He I 4471 is a perfect temperature indicator as the isocontours are almost vertical. At higher temperatures, this line changes into a good diagnostic for the gravity.

$\dot{M}$, the velocity law exponent, $\beta$, and the terminal wind velocity, $v_{\infty}$ (with $\left.v(r) \approx v_{\infty}\left(1-R_{*} / r\right)^{\beta}\right)$, the profile will have a more pronounced emission profile, a steeper red wing or a broader absorption. Note that for lower wind densities, only the core of $\mathrm{H} \alpha$ is refilled by wind emission, and the errors regarding the derived mass-loss rates become larger. We used a "by eye" line profile fitting procedure to find the best-fitting synthetic FASTWIND spectrum for the observed line profiles of each object, to derive their fundamental parameters. The synthetic profiles are convolved with a rotational profile with appropriate $v \sin i$ (cf. Table 3), whereas the macro-turbulence $v_{\text {macro }}$ (well visible in the wings of the Si-lines) is considered as an additional free parameter, determined in parallel with the fit and accounting for a typical radial-tangential distribution (Gray 1978).

In a first step, we derive coarse parameters by using an extensive grid of synthetic models, with effective temperatures ranging from $10000 \mathrm{~K}$ up to $30000 \mathrm{~K}$ (taking steps of $1000 \mathrm{~K}$ ) and appropriate gravities in steps of $0.1 \mathrm{dex}$ in $\log g$ (systematically 
shifting the gravity range to higher values for higher effective temperatures). We consider the grid steps in $T_{\text {eff }}$ and $\log g$ as a rough estimate (see Sect. 5) for their uncertainty. For each $T_{\text {eff }} / \log g$ grid point, six different values (equidistant in the $\log$, with appropriate boundaries) for the wind strength parameter $Q$ have been calculated, with $Q=\dot{M} /\left(v_{\infty} R_{*}\right)^{1.5}$, thus combining the effect of mass loss, terminal wind velocity, and radius into one single parameter. The reason for doing so is that different combinations of these individual parameters, leading to the same $Q$-value, lead to almost identical H $\alpha$ profiles (Puls et al. 1996), whereas the other lines also remain conserved (Puls et al. 2005). So far, the "real" fit quantity is $Q$ and not the mass-loss rate itself, which is determined only after $v_{\infty}$ and $R_{*}$ have been adopted and/or derived.

As a first guess, we adopted a "typical" radius for each grid point, as calculated from evolutionary models, and followed the observed terminal velocities of massive hot supergiants from Prinja \& Massa (1998) to initialise $v_{\infty}$. In combination with the predescribed $Q$-values, this leads to a wide spread in massloss rates. As mentioned above, all models were calculated for the "old" solar silicon abundance. For the grid, we also considered solar values for the helium content $(n(\mathrm{He}) / n(\mathrm{H})=0.10)$. However, these values were adapted whenever required. Finally, all profiles have been calculated for three different values of the micro-turbulent velocity, $v_{\text {micro }}$, namely 5,10 , and $15 \mathrm{~km} \mathrm{~s}^{-1}$.

After having derived a coarse fit by comparison with the synthetic spectra from our model grid, we further refined the stellar and wind parameters (in particular, $\dot{M}$ and $\beta$ ) to obtain the best possible fit. Note that the micro-turbulent velocity was also adapted when necessary. We consider this quantity to be spatially constant and identical for all investigated lines, i.e., we assume that $v_{\text {micro }}$ does not follow any kind of stratification throughout the atmosphere (see Sect. 5).

In a last step, the actual radius (contrasted to the adopted one) was estimated in an iterative process, by comparing the $V$-band integrated theoretical fluxes with corresponding absolute visual magnitudes $M_{V}$, which in turn were taken from the calibrations by Schmidt-Kaler (1982) (except for HD 168183 and HD 111904, which are known cluster members and hence we could derive $M_{V}$ from their distance). This procedure (including corresponding updates of $\dot{M}$ ) was repeated until the difference in input and output radius became negligible (usually one or two iterations were sufficient).

Note that the derived gravities are contaminated by centrifugal effects. To obtain the "true" gravities needed to calculate, e.g., the masses and to find the appropriate positions in the $\log T_{\text {eff }}-\log g$ diagram (Sect. 9), one has to apply a "centrifugal correction" with respect to the derived rotational velocities (Repolust et al. 2004, and references therein). These corrections have been applied, and the corresponding values can be found in Table 6 as entry $g_{\text {corr }}$. For our further analysis, we will use exclusively these data.

\subsection{Results from the line fits}

Due to the restricted number of available spectral lines and because different regimes of the parameter space lead to different accuracy, given the available diagnostic, we subdivide our sample in three groups of stars, depending on the reliability of the derived stellar parameters (mainly $T_{\text {eff }}$ and $\log g$ ). The first group (hereafter "group I") comprises sample stars for which we consider the results to be very reliable. The second group constitutes objects that suffer from the following "defect". From Fig. 3, it is obvious that there will be models at each side of the "peak" around $23000 \mathrm{~K}$ that produce similar Si III 4552 (and 4567-4574) profiles. Since these lines are our major temperature indicator for the early-type stars, this leaves us with two possibilities for $T_{\text {eff }}$, and only the strengths of additional $\mathrm{Si}$ II or Si IV lines would allow for a conclusive answer. For sample stars of spectral type B1-B2, only the Si III multiplet is available to us. In this case, we make the appropriate choice between the high and the low temperature solution relying either on He I 4471 (which still has a certain sensitivity on the temperature, but requires an assumption of the helium abundance) or on its spectral subtype (which we infer from SIMBAD or recent literature). Due to the restricted reliability of this approach, all corresponding objects are collected in "group II". We discuss this approach in detail in the appendix, for the prototypic example of HD 54764, which is a B1 Ib/II star. The effective temperatures and gravities derived for group I and II objects will finally be used to obtain a new calibration of $T_{\text {eff }}$ as a function of spectral subtype. "Group III" contains stars for which we have no means to derive accurate stellar parameters, either because the objects are rather extreme or suffer from additional effects not included in our atmospheric models, either because of their peculiar spectrum that complicates a reliable fit, or a combination of both. Therefore we classify these stars as "unreliable", and their parameters should be considered with caution. Apart from these three groups, we define a fourth group (hereafter "group IV") consisting of the twelve comparison stars. For these objects, at most three lines have been observed (He I 4471, $\mathrm{H} \gamma$, and $\mathrm{H} \alpha$ ), which, in combination with our new $T_{\text {eff-calibration (see above), }}$ will be used to estimate effective temperatures, surface gravities, and wind parameters.

For most of the sample stars we observed two $\mathrm{H} \alpha$ profiles about one year apart, to obtain an impression of the wind variability (for a detailed investigation, see Markova et al. 2005). We model each $\mathrm{H} \alpha$ profile separately by fixing all parameters except for the mass-loss rate. The resulting two values for $\dot{M}$ are finally averaged to compute the wind strength parameter $\log Q$ and the mean wind density that are required for our further investigations in Sect. 10.

In the (electronic) appendix we will display and discuss, where necessary, the individual fits one by one, for all four "reliability groups". A summarising table with the derived stellar and wind parameters of all B type supergiants is presented in Table 6, which is only available in the electronic edition. In the following, we give some comments on general problems encountered during our fitting procedure.

\section{Comments on general problems}

We have noted a certain discrepancy between the two lines of the Si II 4128-4130 doublet. Theory predicts the Si II 4130 line to be somewhat stronger than the Si II 4128 line, since the $g f$ value of the second component is roughly a factor 1.5 larger than of the first component (different atomic databases give very similar results). However, in most (but not all) cases, we observe an equal line strength for both lines. While further investigation is needed, we approached this problem by finding a compromise solution in the final fit. The related errors are discussed in Sect. 5.

Thanks to the high spectroscopic resolution, a problem with the forbidden component in the blue wing of He I 4471 could be identified. It appears that this (density dependent) component is often (however not always) predicted too weak for the early-type stars $(<\mathrm{B} 1)$ and too strong for the late type stars $(>\mathrm{B} 2)$. For the cooler stars, this might be explained by a fairly strong $\mathrm{O}$ II blend at this position. 
For some stars, He I 6678 would need a higher macroturbulence, $v_{\text {macro }}$, than the other lines. The clearest example of this situation is given by HD 92964, but, to a lesser extent, it also occurs in HD 89767, HD 94909, HD 93619, HD 96880, and HD 106343, all of them being early-type stars. Their He I 6678 "fits" show that there must be an additional broadening mechanism that we do not understand at present.

In a number of cases we were not able to reproduce the shape of the $\mathrm{H} \alpha$ profile, mainly for two reasons. On the one hand, the assumption of spherical symmetry adopted in FASTWIND (and almost all other comparable line-blanketed NLTE $\operatorname{codes}^{1}$ ) prohibits the simulation of disks or wind compressed zones in the case of large rotational speeds. On the other hand, we neglected the effects of wind clumping (small-scale density inhomogeneities redistributing the matter into dense clumps and an almost void interclump medium, see, e.g., Puls et al. 2006, and references therein), which can have a certain effect on the shape of the $\mathrm{H} \alpha$ profile and on the absolute value of the mass-loss rate. Recent findings by CR06 have indicated that this effect is rather small in B-type supergiants though. Even if the detailed shape of $\mathrm{H} \alpha$ is not matched, the error in the derived (1D) mass-loss rate remains acceptable, due to the strong reaction of the profile on this parameter, at least if the wind densities are not too low. For such low wind densities then, the discussed processes do not lead to any discrepancy with the observed profile shape, since only the core of $\mathrm{H} \alpha$ becomes refilled.

We stress that, in this kind of analysis, a reliable normalisation of the spectra is of crucial importance. An incorrect normalisation of the silicon lines, for example, leads to errors in the derived effective temperatures, which will propagate into the derived surface gravities ${ }^{2}$. Errors occurring in the normalisation of $\mathrm{H} \gamma$ additionally enlarge the error in $\log g$, whereas an erroneous rectification of $\mathrm{H} \alpha$ affects the $Q$ value and thus the mass-loss rate. Although we were restricted to few selected orders (thus cutting out the largest part of the available continuum), the remaining spectral windows were generally sufficient to obtain a correct normalisation thanks to the high $\mathrm{S} / \mathrm{N}$ ratio that was obtained. For pure emission $\mathrm{H} \alpha$ profiles, on the other hand, this was more difficult, due to the large width of the profiles.

A reliable derivation of terminal velocities, $v_{\infty}$, turned out to be possible only for a restricted number of stars. As already explained, we adopted the values determined by Prinja \& Massa (1998) from the UV as a first estimate, with an extrapolation towards later B-types by using the corresponding A supergiant data provided by Lamers et al. (1995). Both data sets have been collected in Table 1 of Kudritzki \& Puls (2000). By means of these values, for most of the objects such a good fit in $\mathrm{H} \alpha$ (and other lines) had been obtained in the first instance that further alterations seemed to be unnecessary. Only for HD 92964, the adopted $v_{\infty}$-value might be too low, which could explain the mismatch of He I 6678. However, the first $\mathrm{H} \alpha$ profile of this object is in complete emission, and the second one displays only a tiny absorption dip, which makes it difficult to derive a reliable value. Thus, also for this star, we adopted the UV-value.

On the other hand, by alternatively deriving $v_{\infty}$ from fitting the shape of $\mathrm{H} \alpha$, we generally found values that are either similar or lower than the ones from the UV. Though the agreement was extremely good for some objects, for others a discrepancy by more than a factor of two was found, resulting in a mean

\footnotetext{
${ }^{1}$ See Zsargó et al. (2006) for recent progress regarding a 2D modelling.

2 To preserve the $\mathrm{H} \gamma$ profile, changing the temperature by $1000 \mathrm{~K}$ requires a simultaneous change in gravity by roughly 0.1 dex.
}

difference of $\mathrm{H} \alpha$ and UV terminal velocity of $45 \%$. Note that the fits generally improved when adopting the UV values finally. Thus we conclude that it is not possible to precisely estimate $v_{\infty}$ from $\mathrm{H} \alpha$ alone, at least in a large fraction of cases, where the typical error by such an approach is given by a factor of two.

\section{Error estimates}

Thanks to the high quality of our spectra, fitting errors due to resolution limitations or instrumental noise do not play a role in our analysis. The major problem encountered here is the very restricted number of available lines, and the involved assumptions we are forced to apply (particularly regarding the Si abundance and the micro-turbulent velocity, see below). Apart from this principal problem, the major source of errors is due to our "eyefit" procedure (contrasted to automated methods, e.g., Mokiem et al. 2005), which is initiated by manually scanning our precalculated grid (see Sect. 4.2). The effective temperatures and gravities did not need refinement once a satisfactory solution had been found from the grid, after tuning the mass-loss rates and the velocity exponents. Therefore, the grid steps reflect the errors on those parameters. In practice, this means that typical errors are of the order of $1000 \mathrm{~K}$ in $T_{\text {eff }}$ and 0.1 in $\log g$, which is - for later spectral types - somewhat larger than possible under optimal conditions, i.e., if many more lines were available. Finer step sizes or further fine-tuning of the models with respect to effective temperature, on the other hand, was regarded as irrelevant, due to the consequences of our assumptions regarding abundance and micro-turbulence.

\subsection{Error estimates for $T_{\text {eff }}$}

Whenever many lines from one ion are present, the microturbulence can be specified with a high precision given a "known" abundance. A few lines from different ionisation stages, on the other hand, allow for a precise temperature estimate, since in this case the ratios of lines from different stages are (almost) independent of abundance (which is the reason that spectral classification schemes use these ratios). Missing or incomplete knowledge becomes a major source of uncertainty if only a few lines from one ionisation stage are available. We assess the different effects one by one.

\subsubsection{Influence of Si abundance}

Concentrating first on $\mathrm{Si}$, a star with depleted abundance will display, at a given temperature, weaker Si lines in all ionisation stages, and vice versa, if $\mathrm{Si}$ is enhanced. Thus, if the line strengths decrease with increasing temperature (as for Si II, see Fig. 2), the effective temperature would be overestimated if the actual abundance is lower than the assumed solar value, and underestimated for increasing line strength with temperature (e.g., for the low temperature region of Si III, cf. Fig. 3). To check the quantitative consequences of this uncertainty, we calculated, for three different temperatures $(15000,20000$, and $25000 \mathrm{~K})$, various models that are depleted and enhanced in Si by a factor of two (thus comprising the lower values discussed in the literature, see Sect. 4.2), and investigated how much the derived temperature would change. When changing the effective temperature of the model, one has to change the surface gravity in parallel, to preserve the $\mathrm{H} \gamma$ profile.

For late-type stars at $15000 \mathrm{~K}$ (where only Si II is available), such a depletion/enhancement of $\mathrm{Si}$ corresponds to a 
decrease/increase of $T_{\text {eff }}$ by $2000 \mathrm{~K}$ (and $\log g$ by 0.2 ). At $25000 \mathrm{~K}$, which is a representative temperature for the earlytype objects for which we only have the Si III triplet, the effect was found to be identical. At $20000 \mathrm{~K}$, the effect depends on whether we have Si II or Si III at our disposal, and the overall effect is a bit smaller. If we have $\mathrm{Si}$ II, we again find an overestimation, now by $1500 \mathrm{~K}$, if the star is depleted in $\mathrm{Si}$, but assumed to be of solar composition. If we have Si III, which is still gaining in strength in this temperature regime, the effective temperature would be underestimated by $1500 \mathrm{~K}$. In conclusion, due to the uncertainties in the $\mathrm{Si}$ abundance, we expect that our temperature scale might systematically overestimate the actual one (except for those group II objects that rely on Si III, where an underestimation is possible), by $1500 \mathrm{~K}$ to $2000 \mathrm{~K}$ if the average abundance were actually 0.3 dex lower than solar.

\subsubsection{Influence of $v_{\text {micro }}$}

One might argue that a Si depletion is not present in our sample, since in almost all cases our secondary temperature diagnostic, He I, was fitted in parallel with Si without further problems. However, we have no independent check of the He content (only one ion available). In most cases, the He I line profiles were consistent with solar abundance, but strongly evolved objects have processed material and should have a larger He content (see, e.g., the corresponding discussion in CR06).

Even if one would regard the consistency between $\mathrm{Si}$ and $\mathrm{He}$ as conclusive, it depends on one of our additional assumptions, namely that the micro-turbulent velocities are constant with height, i.e., identical for $\mathrm{He}$ and $\mathrm{Si}$ lines $^{3}$. Although there is no clear indication in the present literature that this hypothesis is wrong, a stratified micro-turbulent velocity seems plausible. In such a case (i.e., different $v_{\text {micro }}$ for $\mathrm{He}$ and $\mathrm{Si}$ ), we would no longer have a clear handle on this quantity, and due to the well-known dependence of line strength on this quantity (for Si lines, see, e.g., Trundle et al. 2004; Urbaneja 2004; for He lines, McErlean et al. 1998), an additional source of error would be present. From test calculations, it turned out that a change of $1000 \mathrm{~K}$ (which is our nominal error in $T_{\text {eff }}$ ) corresponds to a change of $v_{\text {micro }}$ by roughly 4 to $5 \mathrm{~km} \mathrm{~s}^{-1}$ in the Si lines.

For the few objects with low macro-turbulent velocity, $v_{\text {macro }}$, and low rotational speed, we were able to directly "measure" $v_{\text {micro }}$, thanks to the high resolution of our spectra. In these cases, the profiles become too narrow in the wings and too strong in the core, when decreasing $v_{\text {micro }}$ (and vice versa when increasing $v_{\text {micro }}$ ). This behaviour cannot be compensated for by changing $T_{\text {eff }}$. For most of the objects, however, such precise information is washed out by $v_{\text {macro }}$. In the majority of cases we were able to obtain satisfactory fits ( $\mathrm{He}$ in parallel with $\mathrm{Si}$ ) by keeping typical values available in our grid, which are $15 \mathrm{~km} \mathrm{~s}^{-1}$ for early-type objects and $10 \mathrm{~km} \mathrm{~s}^{-1}$ for late-type objects. Changing $v_{\text {micro }}$ by more than 2 to $3 \mathrm{~km} \mathrm{~s}^{-1}$ would destroy the fit quality of either Si or He. In conclusion, we are confident about the derived values of $v_{\text {micro }}$ (and thus of the temperatures), provided that the He and Si lines are affected by a similar micro-turbulent broadening, i.e., that stratification effects are negligible.

${ }^{3}$ For most of our objects, the analysed He lines are stronger than the $\mathrm{Si}$ lines, i.e., they are formed above the $\mathrm{Si}$ lines.

\subsubsection{Influence of the Si II problem}

As mentioned in Sect. 4.3, there is a discrepancy between the two lines of the Si II 4128-4130 doublet for most of our latetype objects. By allowing for a compromise solution (in which Si II 4128 is predicted as too weak and Si II 4130 as too strong), we minimise the error. Indeed, to fit either of the two lines perfectly, we would have to change the effective temperature by roughly $500 \mathrm{~K}$, which is well below our nominal error.

\subsection{Error estimates for other quantities}

Although the fit-/modelling-error in $\log g$ is \pm 0.1 for a given effective temperature, $\log g$ itself varies with $T_{\text {eff }}$ (as already mentioned, typically by 0.1 for $\Delta T=1000 \mathrm{~K}$ ), so that for a potentially larger error in $T_{\text {eff }}$ (due to under-/overabundances of $\mathrm{Si}$ ) the gravity also has to be adapted. The errors for the other parameters follow the usual error propagation (for a detailed discussion, see Markova et al. 2004; and Repolust et al. 2004), being mainly dependent on the uncertainty in the stellar radius, which in turn depends on the validity of the used calibration of the absolute visual magnitude from Schmidt-Kaler (1982). A precise error estimate of the latter quantity is difficult to obtain, but at least for early and mid Ia supergiants (until B3 Ia) we can compare this calibration with the results from CR06 (Sect. 2), who derived $M_{V}$ values either directly from cluster membership arguments or from average subtype magnitudes of Magellanic Cloud stars. Comparing their results with ours, we find similar average values, with a $1-\sigma$ scatter of

$\Delta M_{V} \approx \pm 0.43 \mathrm{mag}$

which will be adopted in the following, also for the later spectral types and the other luminosity classes. From this number and the error in $T_{\mathrm{eff}}$, the error in the radius becomes

$\Delta \log R_{*} / R_{\odot} \approx \pm 0.088$,

which corresponds to $22 \%$, and is consistent with the differences in the radii derived by us and by CR06, see also Table 4 . We subsequently find a typical uncertainty in the luminosity of

$\Delta \log L / L_{\odot} \approx 0.22 \ldots .0 .19$

for $T_{\text {eff }}=12000 \mathrm{~K} \ldots 25000 \mathrm{~K}$, respectively. The wind-strength parameter, $\log Q$, can be determined with rather high precision. Adopting a combined contribution of fit error and uncertainty in $T_{\text {eff }}$ of \pm 0.05 (Repolust et al. 2004, their Sect. 6.2), and an additional contribution of \pm 0.1 accounting for the temporal variability (Table 6 and Sect. 10), we find

$\Delta \log Q \approx \pm 0.11$.

The precision in $\Delta \log \dot{M}$ amounts to

$\Delta \log \dot{M} \approx \pm 0.24$,

if we estimate the error in $v_{\infty}$ as $30 \%$. Finally, the error in the derived wind-momentum rate is

$\Delta \log D_{\text {mom }} \approx \pm 0.34$,

i.e., somewhat larger than the error in $\log L$.

\section{Comparison with other investigations}

We compare the results of our analysis with corresponding ones from similar investigations, in particular those by CR06, McErlean et al. (1999), and Kudritzki et al. (1999) for five stars in common. 
Table 4. Comparison between the fundamental parameters derived in this study and by CR06, McErlean et al. (1999), and Kudritzki et al. (1999) for the objects in common.

\begin{tabular}{ccccccccccl}
\hline \hline HD & $\begin{array}{c}T_{\text {eff }} \\
(\mathrm{kK})\end{array}$ & $\begin{array}{c}\log g \\
(\mathrm{cgs})\end{array}$ & $M_{V}$ & $\begin{array}{c}R_{*} \\
\left(R_{\odot}\right)\end{array}$ & $\log L / L_{\odot}$ & $\begin{array}{c}v_{\infty} \\
\left(\mathrm{km} \mathrm{s}^{-1}\right)\end{array}$ & $\beta$ & $\log Q$ & $\begin{array}{c}v_{\text {micro }} \\
\left(\mathrm{km} \mathrm{s}^{-1}\right)\end{array}$ & Reference \\
\hline \multirow{2}{9}{94909} & 25.0 & 2.7 & -6.9 & 36 & 5.65 & 1450 & 1.8 & -12.96 & 20 & this study \\
& 27.0 & 2.9 & -6.4 & 25.5 & 5.49 & 1050 & 1.5 & -12.34 & 10 & CR06 \\
91943 & 24.0 & 2.7 & -5.95 & 23 & 5.19 & 1400 & 2.5 & -13.36 & 15 & this study \\
& 24.5 & 2.8 & -6.3 & 26.8 & 5.35 & 1470 & 1.2 & -13.01 & 10 & CR06 \\
148688 & 21.0 & 2.5 & -6.9 & 42 & 5.49 & 1200 & 3.0 & -12.90 & 15 & this study \\
& 22.0 & 2.6 & -6.8 & 36.7 & 5.45 & 725 & 2.0 & -12.39 & 15 & CR06 \\
53138 & 17.0 & 2.15 & -7.0 & 50 & 5.27 & 490 & 2.5 & -13.20 & 10 & this study \\
& 15.5 & 2.05 & -7.3 & 65 & 5.34 & 865 & 2.0 & -13.57 & 20 & CR06 \\
& 18.5 & 2.35 & & & 5.04 & & & & 10 & McErlean et al. (1999) \\
58350 & 18.5 & 2.30 & & 39.6 & 5.22 & 620 & 2.5 & -13.61 & 40 & Kudritzki et al. (1999) \\
& 13.5 & 1.75 & -7.0 & 65 & 5.10 & 250 & 2.5 & -13.17 & 12 & this study (group IV) \\
& 16.0 & 2.10 & & & 5.36 & & & & 15 & McErlean et al. (1999) \\
\hline
\end{tabular}

\subsection{Comparison with the analysis by Crowther et al. (2006)}

Our sample has four targets in common with the sample of Galactic early B supergiants studied by CR06: HD 94909 (B0 Ia), HD 91943 (B0.7 Ib), HD 148688 (B1 Ia), and HD 53138 (B3 Ia). They used the alternative NLTE model atmosphere code CMFGEN (Hillier \& Miller 1998) to derive the physical parameters and wind properties of these stars. Compared to their low dispersion CTIO and intermediate dispersion JKT/INT spectra, we have the advantage of the very high resolution CES data. On the other hand, CR06 have complete spectra at their disposal (kindly provided to us by P. Crowther). To compare the spectra with each other, we first downgraded our synthetic spectral lines by convolving with an appropriate Gaussian. Subsequently, we verified whether the best model we found from a limited number of lines also provides a good fit to the additional $\mathrm{H}, \mathrm{He}$, and $\mathrm{Si}$ lines in the complete spectrum. CR06 used a solar Si abundance as well and 0.20 by number for the helium abundance. The complete comparison is summarised in Table 4. In this procedure, we only re-determined the mass-loss rates from the low/intermediate-resolution spectra of CR06 to fit the $\mathrm{H} \alpha$ profile. They do not differ significantly from the values derived from our data.

HD 94909 (B0 Ia). From the CTIO spectrum of this star, CR06 estimated the effective temperature to be $27000 \mathrm{~K}$ and $\log g$ 2.9. It is impossible to fit our Si III lines with such a high temperature, because they become far too weak. Instead, we derive an effective temperature of $25000 \mathrm{~K}$, in combination with a $\log g$ of 2.7. When we compare our (degraded) best-fitting model with the CTIO spectrum (Fig. 5a), we see that Si IV is predicted too weak, which explains the higher temperature found by CR06. By exploring the neighbouring parameter space, it turned out that we cannot simultaneously fit Si III and $\mathrm{Si} \mathrm{IV}$, and we suggest that this star is overabundant in $\mathrm{Si}$.

HD 91943 (B0.7 Ib). For this star, only a few additional lines (besides those measured by us) are available due to the high noise level. In Fig. 5b we show Si III 4552-4567-4574 for consistency, together with lines of an additional ionisation stage of Si (Si II 4128 and Si IV 4089/4116/4212) and helium (He I 4026, He I 4387, and He II 4200). There might be a problem with the normalisation of the Si IV 4212 and Si IV 4089 profiles, but still it is clear that the strength of the observed and theoretical profiles agree satisfactorily. In view of the low dispersion, we obtain a reasonable fit, which gives us confidence in our results.

HD 148688 (B1 Ia). This star is often used as a comparison star in UV studies as a galactic counterpart for early B-type supergiants in M31, M33, or the SMC (Bresolin et al. 2002; Urbaneja et al. 2002; Evans et al. 2004, respectively). It is one out of a few that show no radial-velocity changes. We have only a few additional lines available in the CTIO spectrum. It is very encouraging that they all nicely confirm our results (see Fig. 5c). To fit the Si III triplet, we need an effective temperature of $1000 \mathrm{~K}$ lower than the one suggested by CR06.

HD 53138 (B3 Ia) can surely be named one of the most "popular" B-type supergiants studied until now. Let us first concentrate on our high resolution spectrum (Fig. A.3. in the Appendix). Clear variations in the wind outflow are registered. In the first measurement of $\mathrm{H} \alpha$, the $\mathrm{P}$ Cygni profile has only a tiny absorption trough and a considerable emission, whereas the second profile indicates a much lower wind density. This star is one of the objects to show the discrepancy between predicted and actual line strength of the Si II doublet components (Sect. 4.3) for which we adopted a compromise solution. In this way, our bestfitting model gives an effective temperature of $18000 \mathrm{~K}$ and a $\log g$ of 2.25 .

When degrading the resolution of this best-fitting model to the resolution of the full spectrum provided by CR06 (originating from the LDF Atlas ${ }^{4}$ ), we find some discrepancy in the Si III triplet (see Fig. 5d). This discrepancy can be resolved by decreasing the temperature in combination with either a lower micro-turbulent velocity or a depletion in Si, since Si IV 4089 also seems to be a bit too strong. To cure this problem, we can go down as far as $17000 \mathrm{~K}$ (with $\log g=2.15$ and $v_{\text {micro }}=$ $10 \mathrm{~km} \mathrm{~s}^{-1}$ ), which is still $1500 \mathrm{~K}$ higher than the value derived by CR06 $\left(T_{\text {eff }}=15500 \mathrm{~K}, \log g=2.05\right)$. To find an explanation for this difference, we had a closer look at their spectral line fits. Though the overall fit is good, the Si lines are not matched perfectly. In particular, at their value of $T_{\mathrm{eff}}=15500 \mathrm{~K}$, the $\mathrm{Si}$ II 4128-4130 profiles are predicted too strong, whereas Si III 4552-4567-4574 is predicted too weak. This disagreement in the ionisation balance suggests that the effective temperature should be somewhat higher. We use the $17000 \mathrm{~K}$ model, which

\footnotetext{
4 http://www .ast. cam.ac.uk/STELLARPOPS/hot_stars/ spectra_lib/mw_library/mw_library_index.html
} 

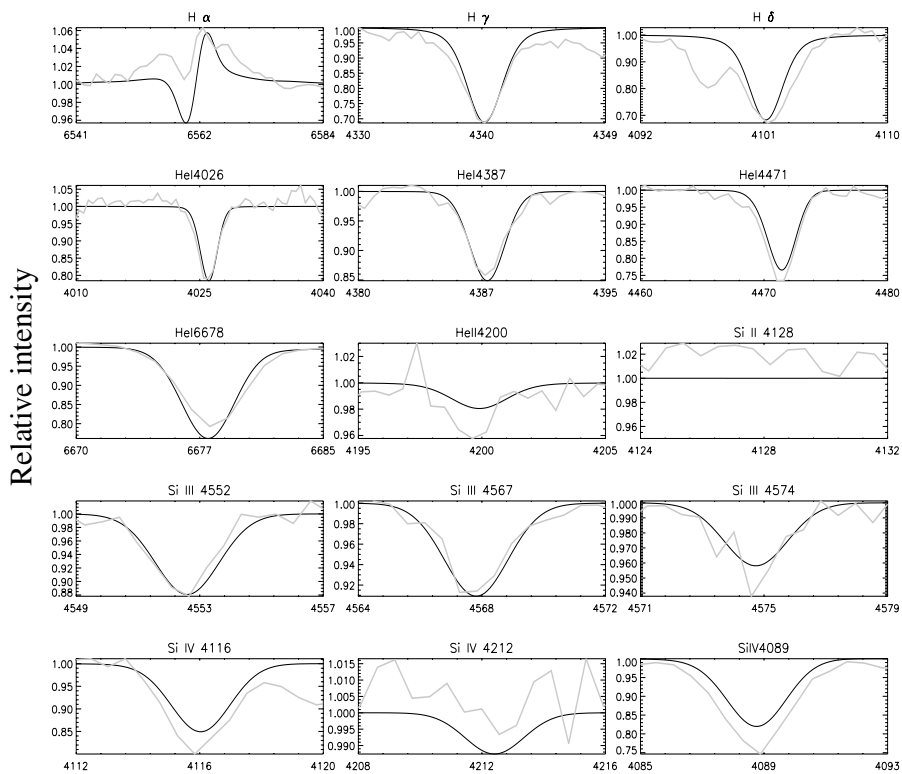

(a)
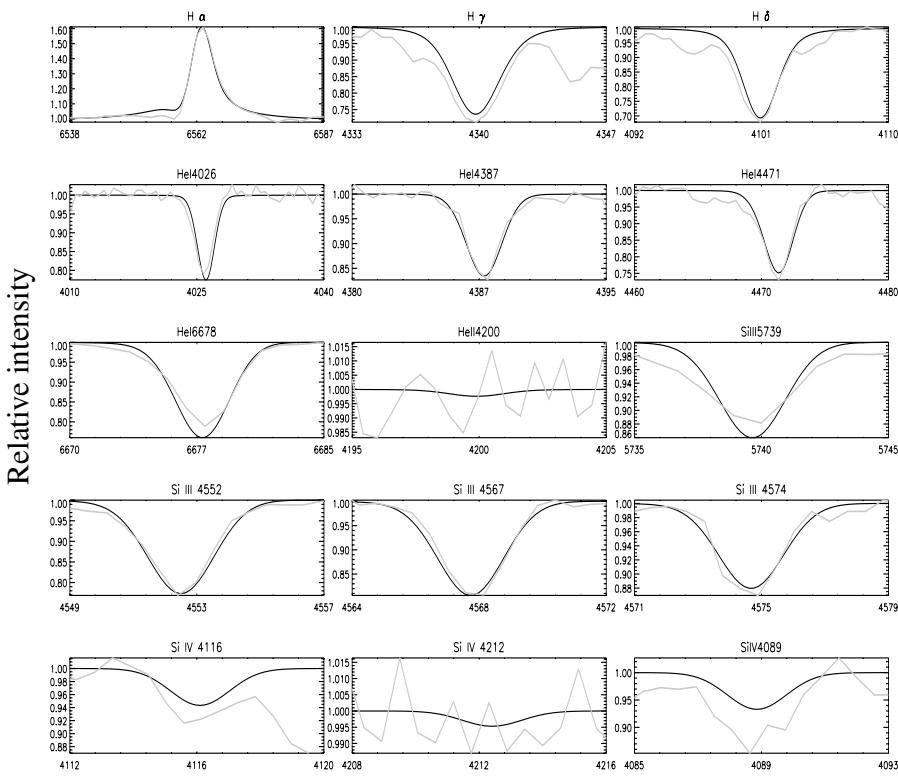

(c)
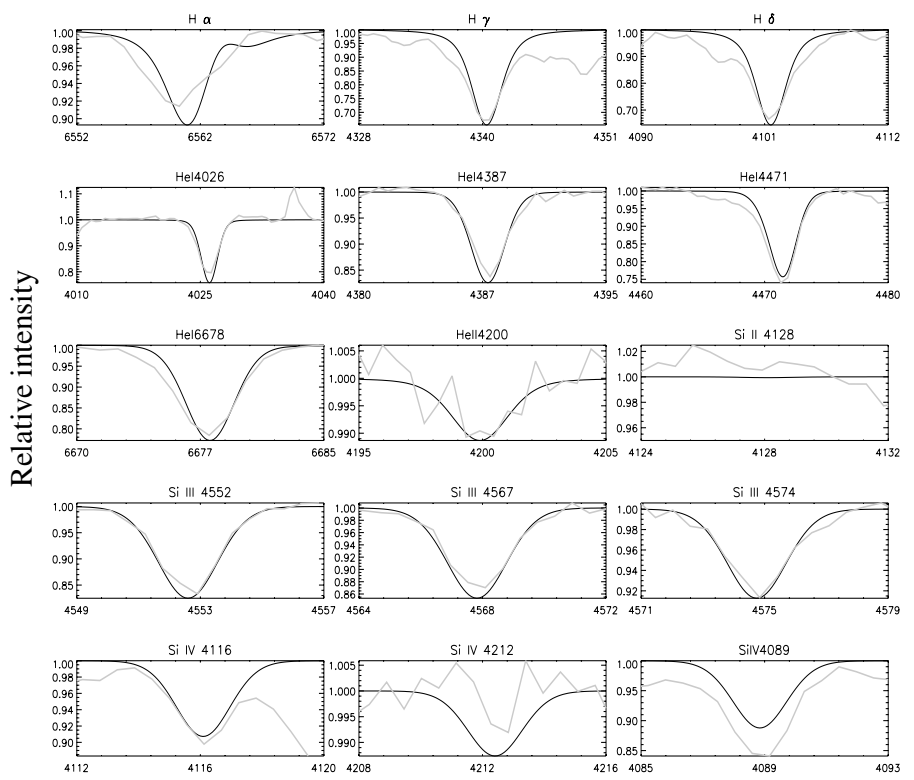

(b)
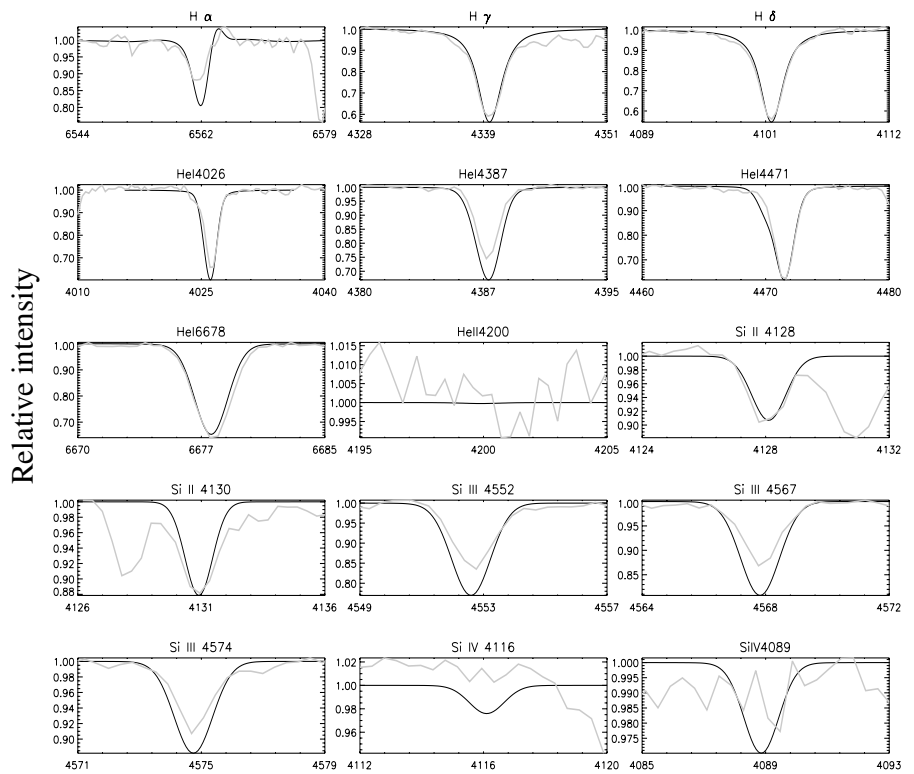

(d)

Fig. 5. Comparison between synthetic line profiles (downgraded) from our best-fitting models (black) and the low/intermediate dispersion spectra of CR06 (grey), for four early B-type supergiants in common: a) HD 94909; b) HD 91943; c) HD 148688; d) HD 53138.

is at the lower bound of the quoted error range of our original analysis, as a solution to all discussed problems.

A summary of the main parameters resulting from both studies is given in Table 4. Interestingly, in all cases, the low/intermediate resolution $\mathrm{H} \alpha$ profile observed by CR06 lies amidst our two $\mathrm{H} \alpha$ profiles, so that the inferred mass-loss rates are very similar and the variability is not large (cf. Sect. 10). As already pointed out, the differences in radii obtained by us and CR06 are of similar order. On the other hand, the $\beta$-values implied by our fits are generally larger than the ones from CR06. Accounting additionally for the moderate differences in $T_{\text {eff }}$ for the first three objects (in these cases, our values are lower), this explains the lower $\log Q$ values that we found. For HD 53138, on the other hand, the $\beta$ values are similar, whereas our effective temperature is larger, explaining the higher $\log Q$ value.

\subsection{More comparisons}

By means of the plane-parallel, hydrostatic, NLTE atmosphere code TLUSTY (Hubeny \& Lanz 2000; McErlean et al. 1999 deduced the photospheric parameters and CNO abundances of 46 Galactic B supergiants. Effective temperatures were mostly obtained from the ionisation balance of Si. Two of their objects are in common with our sample (HD 53138 and HD 58350, a B5Ia Group IV object, see Appendix B.5), and Table 4 displays the comparison. The effective temperature for HD 53138 from McErlean et al. (1999) compares well with our high temperature solution for this object, i.e., differs significantly from the much lower value derived by CR06 (see above), and also the gravities are consistent. Regarding HD 58350, we have adopted a temperature consistent with the calibrations provided in the 
next section, which is significantly lower than the value found by McErlean et al. (1999). For details, we refer to Appendix B.5, but we point out that the complete $J K T$ spectrum allows for an increase in temperature by roughly $1000 \mathrm{~K}$.

Finally, on the basis of the temperature scale derived by McErlean et al. (1999), Kudritzki et al. (1999) analysed the wind properties of a sample of early/mid B and A supergiants, by means of a previous, unblanketed version of FASTWIND. Their value for the wind-strength parameter of HD 53138 coincides with the value provided by CR06, i.e., is lower than our result. We regard this agreement/disagreement as not conclusive, since (i) the $\mathrm{H} \alpha$ spectra used by Kudritzki et al. (1999) are different (less emission), and (ii) the analysis was based on unblanketed models, which in certain cases can lead to differences in the derived mass-loss rate (cf. Repolust et al. 2004, their Fig. 21). A further, more comprehensive comparison will be given in Sect. 8, with respect to modified wind-momenta. Summarising the results from this comparative study, we conclude that, despite the multidimensional parameter space we are dealing with and the interdependence of the parameters, we are able to derive rather accurate values from only a few selected lines (of course, within our assumptions, in particular regarding the $\mathrm{Si}$ abundance). This enables us to provide (and to use) new calibrations for the effective temperatures, which will be discussed in the next section.

\section{7. $T_{\text {eff }}$ calibration and group IV objects}

Thanks to the (almost) complete coverage of the B star range and the large number of objects available, we are able to derive a $T_{\text {eff }}$ calibration as a function of spectral type, which we subsequently use to derive the temperatures of our group IV objects. The spectral types were taken from the literature. In case different assignments were given, we have provided arguments why we prefer one above the other, in the Appendix. Ideally, one would want to re-assign spectral types from our high-quality data as in, e.g., Lennon et al. (1993). However, one needs a fair number of spectral lines to do this in a safe way. Since we have only a few $\mathrm{H}$, $\mathrm{He}$, and $\mathrm{Si}$ lines, we preferred to use the spectral classifications from the literature, keeping in mind that some of them may not be very refined. We confirmed or adopted the luminosity class on the basis of the strength of $\mathrm{H} \alpha$.

In Fig. 6 we see that the effective temperature follows an systematic decrease with spectral type. To derive the temperature calibration, we joined our results for group I/II objects with those from CR06 (who used assumptions similar to ours in their analysis) and added two more objects from Przybilla et al. (2006) at the low temperature edge. In contrast to the errors inherent to our analysis ${ }^{5}$, which are identical to those from CR06, these two objects (HD $34085=\beta$ Ori (B8 Iae:), HD 92207 (A0 Iae)) could be analysed in a very precise way by exploiting the complete spectrum, with resulting errors of only $\pm 200 \mathrm{~K}$.

By performing a polynomial fit to these data (including the quoted errors), we derive the following effective temperature scale for B-type supergiants,

$T_{\text {eff }}=26522.63-5611.21 x+817.99 x^{2}-42.74 x^{3}$,

with $x$ the spectral subclass. Note that we have included both luminosity subclasses (Ia and Ib) to obtain this fit, and that the inclusion of the objects by CR06 changed the results only marginally compared to a regression using our and Przybilla et al.'s (2006) data alone. The obtained standard error for this regression is $\pm 1500 \mathrm{~K}$.

${ }^{5}$ Generally, $\Delta T=1000 \mathrm{~K}$, except for the two B5 objects with $\Delta T=$ $2000 \mathrm{~K}$ (see below).

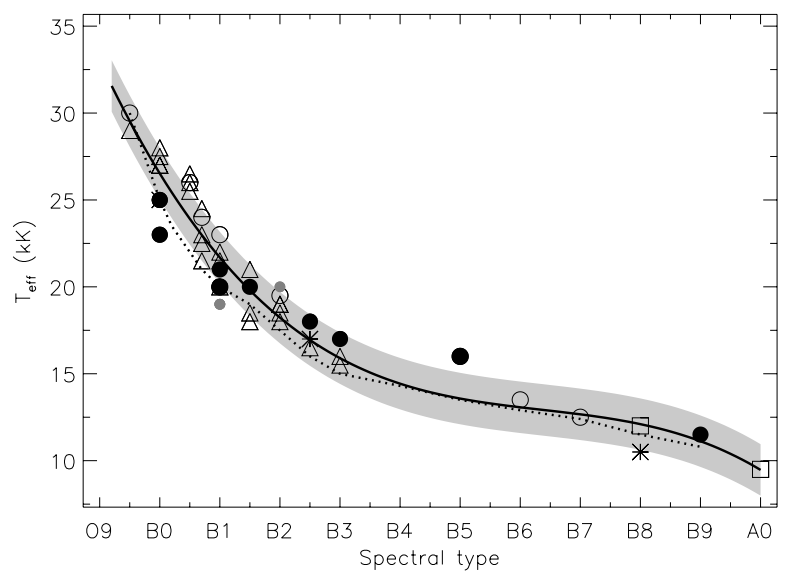

Fig. 6. $T_{\text {eff }}$ as a function of spectral type for the sample B-type supergiants: group I (black circles), group II (small grey filled circles), and group III (asterisks). For group I, we subdivided according to luminosity class: Ia (filled symbols) and Ib (open symbols). Triangles denote the early B Ia supergiants from CR06, and rectangles two late-type Ia supergiants with very precise parameters from Przybilla et al. (2006). The dotted line represents the effective temperature scale of Lennon et al. (1993) and the full line shows our newly derived effective temperature scale based on group I/II stars in addition to the objects from CR06 and Przybilla et al. (2006). The grey area denotes the standard deviation of our regression fit.

When we consider the three group III objects in this figure, they match our derived calibration perfectly although we considered their parameters to be unreliable. The B0 star HD 105056 lies at exactly the same position as HD 94909, just at the lower edge of our error bar. HD 68161 (B8 Ib/II?) will be excluded from our further analysis, due to problems regarding its classification (see Appendix B.4).

As the Ia supergiants have, because of their larger luminosity, more mass loss than Ib objects, they suffer more from line blanketing and mass-loss effects. This is why supergiants of luminosity class Ia are expected to appear cooler than the ones with luminosity class Ib. Comparing the two in Fig. 6, this is indeed exactly what we observe. Our temperature scale agrees well with the one provided by Lennon et al. (1993). The largest differences occur between B0 and B2, where our scale lies roughly $1500 \mathrm{~K}$ higher, mostly due to the objects analysed by CR06.

Let us point out one problem concerning the two B5 objects (with identical $T_{\text {eff }}$ ). We see in Fig. 6 that both temperatures lie clearly above the errors of the calibration. We can still fit the Si II lines well by decreasing the temperature (from $16000 \mathrm{~K}$ to $14500 \mathrm{~K}$ ), when we reduce $v_{\text {micro }}$ by $4 \mathrm{~km} \mathrm{~s}^{-1}$ (see Sect. 5.1.2), which would still be acceptable for these late-type objects. However, with such a low value, a simultaneous fit to the helium and $\mathrm{Si}$ lines becomes impossible, violating our general fitting strategy. Thus, if the lower temperature would be the actual one, this might be due to two reasons, outlined already in Sect. 5: either He and Si have different micro-turbulent velocities, or $\mathrm{Si}$ is underabundant in both B5 targets. Note that the calibration itself remains rather unaffected by these objects, since we used larger error estimates of $\Delta T=2000 \mathrm{~K}$ here.

Finally, by means of this calibration (for particular values, see Table 5), we are able to derive the fundamental parameters for the group IV comparison objects. Using the He I 4471 line as a double check for a consistent effective temperature, the surface gravity is obtained from $\mathrm{H} \gamma$ and the wind properties from $\mathrm{H} \alpha$. Corresponding comments are given in Appendix B.5, and all results are summarised in Table 6 . 

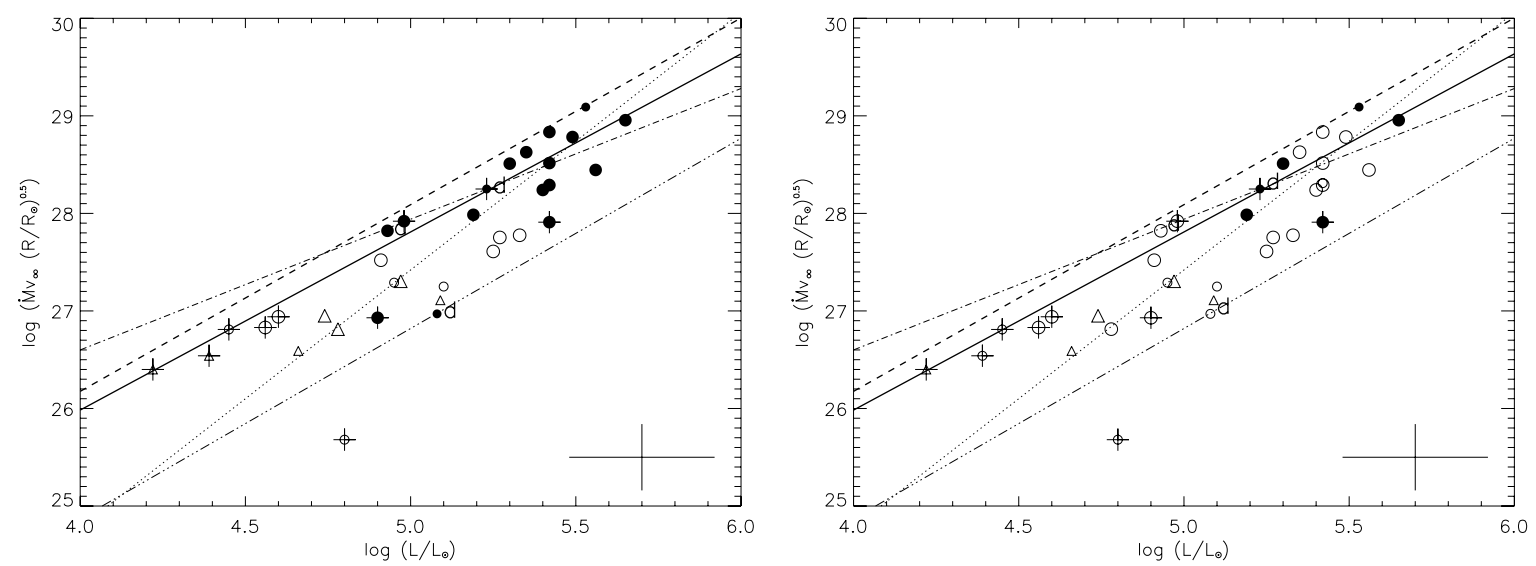

Fig. 7. Wind momentum-luminosity relation (WLR) for our sample of B supergiants. Only class I, II (large symbols) and IV objects (small symbols) have been included, and the mass-loss rates from both $\mathrm{H} \alpha$ profiles were averaged. Bold solid/dashed: theoretical predictions from Vink et al. (2000) for objects with $T_{\text {eff }}>23000 \mathrm{~K}$ and $12500 \mathrm{~K}<T_{\text {eff }}<230000 \mathrm{~K}$, respectively. Dashed-dotted, dashed-dotted-dotted, and dotted lines are the "observed" relations from Kudritzki et al. (1999), for early and mid B supergiants and A supergiants, respectively. Left: results from this study, objects denoted as a function of spectral type. Filled circles: B0/1; open circles: B2... B5; triangles: B6 and later. Right: as left, but objects denoted as a function of $T_{\text {eff }}$. Filled circles: $T_{\text {eff }}>23000 \mathrm{~K}$; open circles: objects with $12500 \mathrm{~K}<T_{\text {eff }}<23000 \mathrm{~K}$; triangles: $T_{\text {eff }}<12500 \mathrm{~K}$. Note that the theoretical bi-stability jump is predicted at $23000 \mathrm{~K}$. Typical error bars are indicated in the lower right. Overplotted crosses denote objects with $\mathrm{H} \alpha$ in absorption, which have a larger error in $\log Q$ than the other objects, due to the uncertainty regarding $\beta$; "d" denotes three objects with disk-like features (HD 47240, HD 64760, and HD 157246).

Table 5. Effective temperature calibration for B-supergiants, based on the results from Sect. 7, used to derive the effective temperatures for the group IV objects.

\begin{tabular}{lc|lc}
\hline \hline SpT & $T_{\text {eff }}$ & SpT & $T_{\text {eff }}$ \\
\hline O 9.5 & 29500 & B 3 & 15800 \\
B 0 & 26500 & B 4 & 14400 \\
B 0.5 & 23900 & B 5 & 13500 \\
B 1 & 21600 & B 6 & 13000 \\
B 1.5 & 19800 & B 7 & 12600 \\
B 2 & 18200 & B 8 & 12100 \\
B 2.5 & 16900 & B 9 & 11100 \\
\hline
\end{tabular}

\section{Wind momentum-luminosity relation}

In Fig. 7 we present the position of our galactic B supergiants in the wind momentum-luminosity diagram, where the modified wind momentum rate is defined as $D_{\text {mom }}=\dot{M} v_{\infty}\left(R_{*} / R_{\odot}\right)^{0.5}$. The presence of such a relation (with wind momenta being a power law of luminosity, and exponents depending on spectral type and metallicity) is a major prediction of the theory of line-driven winds (for details, see, e.g., Kudritzki \& Puls 2000), and has been used in recent years to check our understanding of these winds. To compare our results with earlier findings, we provide results from different investigations relevant in the present context. In particular, the theoretical predictions from Vink et al. (2000) for objects with $T_{\text {eff }}>23000 \mathrm{~K}$ and $12500 \mathrm{~K}<T_{\text {eff }}<23000 \mathrm{~K}$, respectively, are displayed, where the difference is related to an almost sudden change in the ionisation equilibrium of $\mathrm{Fe}$ around $T_{\mathrm{eff}} \approx 23000 \mathrm{~K}$ (from Fe IV to Fe III), the so-called bi-stability jump (Vink et al. 2000, and references therein). Due to this change and below this threshold, the line acceleration is predicted to increase in the lower and intermediate wind, because of the increased number of driving lines being available. Three distinctive lines refer to the findings from Kudritzki et al. (1999), who derived these relations from observed wind momenta of early B, mid B, and A supergiants, respectively.
The wind momenta of our sample objects have been overplotted, by using averaged mass-loss rates (from our two $\mathrm{H} \alpha$ profiles, see also Sect. 10), for group I/II and IV objects. Objects with disk-like features are indicated by "d", and typical error bars are displayed in the lower right. Finally, we have noted objects with $\mathrm{H} \alpha$ in absorption, to indicate that the wind momenta of these objects are subject to errors somewhat larger than typical, of the order of 0.3 dex, due to problems in deriving reliable values for the velocity field exponent, $\beta$ (cf. Puls et al. 1996; and Markova et al. 2004). The left and right figure allow us to compare our findings with the displayed predictions and observations, both for objects as a function of spectral type (left) and as a function of their position with respect to the predicted bistability jump (right).

First, let us point out that differences in their behaviour as a function of luminosity class are minor, and that there is no obvious difference between group I/II and group IV objects, i.e., the wind-momenta for periodic pulsators and comparison objects behave similarly. From the left figure then, we see the following: The position of the early B-types (B0/1) is consistent with both the theoretical predictions and the findings from Kudritzki et al. (1999), except for two objects with uncertain positions and one object with disk-like features. Late B-type supergiants ${ }^{6}$ (B6 and later) nicely follow the observed relation for A supergiants, but are located below theoretical predictions. Only for mid B supergiants (B2...5), we find a difference to earlier results. Whereas Kudritzki et al. (1999) have derived a very strict relation for all B2/3 supergiants of their sample, located considerably below the relation for early-type objects (a finding that still lacks theoretical explanation), our sample follows a non-unique trend. Though high luminosity objects (with $\log L / L_{\odot}>5$ ) behave similarly to the sample studied by Kudritzki et al. (1999) (with a somewhat smaller offset), lower luminosity objects follow the (theoretical) hot star trend, but might also be consistent with the low $T_{\text {eff }}$ relation when accounting for the larger errors in $D_{\text {mom }}$ ( $\mathrm{H} \alpha$ absorption objects).

\footnotetext{
${ }^{6}$ Note that this is the first investigation with respect to this class of objects.
} 
The right figure displays our sample objects as a function of $T_{\text {eff }}$, differentiating for objects with $T_{\text {eff }}>23000 \mathrm{~K}$ and objects with $12500 \mathrm{~K}<T_{\text {eff }}<23000 \mathrm{~K}$, i.e., with temperatures below and above the predicted bi-stability jump. Cooler objects with $T_{\text {eff }}<12500 \mathrm{~K}$ (no predictions present so far) are indicated by triangles. The situation is similar to that above: almost all hotter objects follow the predicted trend, and also a large number of cooler objects follows this trend or the alternative one. Additionally, however, there are a number of cool objects that lie below both predictions, particularly at intermediate luminosities with $4.7<\log L / L_{\odot}<5.4$.

Interestingly, there is only one "real" outlier, an $\mathrm{H} \alpha$ absorption object of group IV (HD 157038), which is an evolved star that is being significantly enriched in $\mathrm{He}$ and $\mathrm{N}$. Note that the three objects with disk-like features display a rather "normal" wind-momentum rate, though one of those (HD 157246) lies at the lower limit of the complete sample. Nevertheless, we will omit the latter stars (as well as group III objects) from all further analyses regarding mass loss to obtain clean results.

Summarising the results from above, no obvious differences compared to earlier findings could be identified within our complete sample (pulsators and comparison objects), with the only exception being that the unique trend for mid B-type supergiants claimed by Kudritzki et al. (1999) could not be confirmed by us: Part of these objects seem to behave "normally", whereas another part shows a momentum deficit. Note also that our findings are not contradictory to those of CR06 (keeping in mind that their sample only included B supergiants not later than B3).

\section{Position in the HR and $\log T_{\text {eff- }}$ - $\log g$-diagram}

After having derived the atmospheric parameters with satisfactory precision, we are now in a position to tackle the major objective of this paper, namely to try and clarify if the opacity mechanism could be held responsible for the variability of our targets, as proposed by Waelkens et al. (1998) and Aerts (2000a). To do so, we calculated the luminosities of the targets from the effective temperatures and stellar radii derived from our analysis. When comparing the relative difference in temperature and luminosity between our values and those derived by Waelkens et al. (1998), we notice that, in general, effective temperatures agree fairly well, though a trend towards lower spectroscopic values is visible. The typical differences are less than $5 \%$ in logarithmic scale, with a maximal difference of $10 \%$. On the other hand, the changes in luminosity are significant (up to $40 \%$ in the $\log$, see Fig. 8).

Since the temperatures agree so well, the luminosity difference is mainly due to the difference in stellar radii. As discussed in Sect. 5.2, the typical errors of our luminosities are of the order of $\Delta \log L \approx 0.19 . .0 .22$, so that at least half of the displayed difference should be attributed to problems with the values provided by Waelkens et al. (1998). This is not surprising, since these authors used photometric calibrations for main-sequence stars because they had no spectroscopic data available. We are confident that our values are more trustworthy, and that the estimated error bars are reliable.

In Fig. 9 we compare the position of the variable B-type supergiants in the HR diagram from spectroscopic and photometric derivations and place them among known variable supergiants available in the literature. It shows that the new position of the sample supergiants is quite different from the one obtained by Waelkens et al. (1998). The stars now lie at the lowluminosity region of the known $\alpha$ Cyg variables. Also shown are the theoretical instability strips of opacity-driven modes in

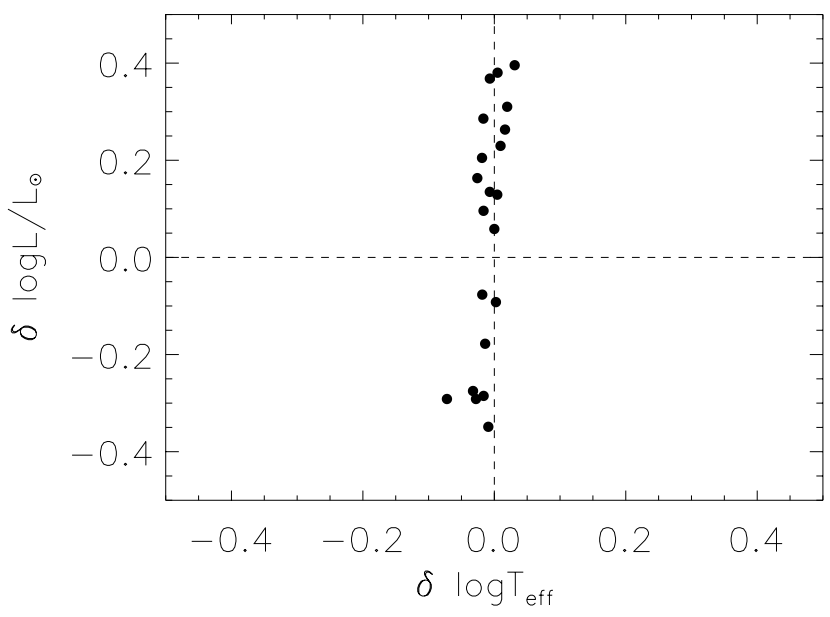

Fig. 8. The relative difference in luminosity against the relative difference in effective temperature between photometrically (Waelkens et al. 1998) and spectroscopically (this paper) determined stellar parameters (with the relative difference defined as $\left.\delta x=\left(x_{\text {Lefever }}-x_{\text {Waelkens }}\right) / x_{\text {Lefever }}\right)$.

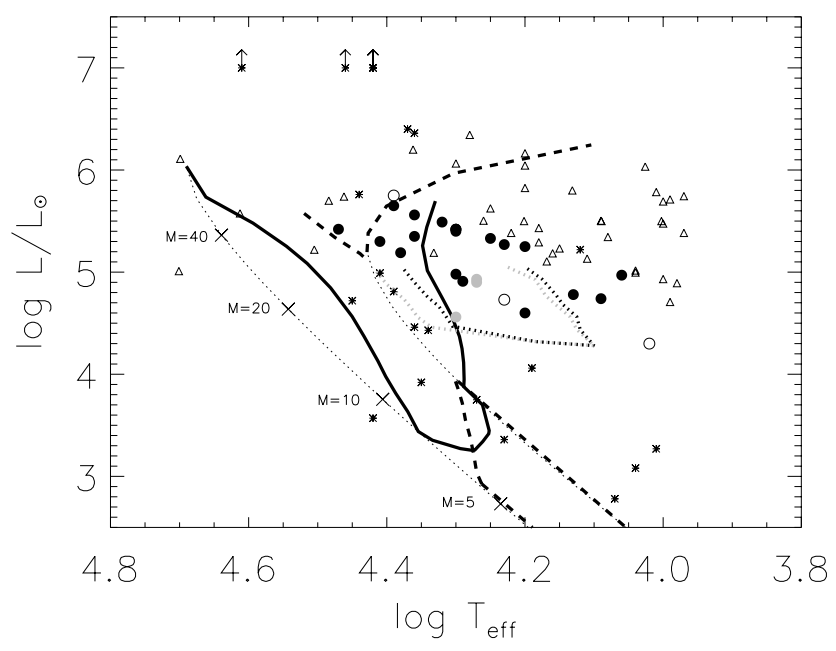

Fig. 9. Position of the sample stars in the HR diagram. Open triangles indicate some well-studied periodically variable supergiants reported in the literature (Burki 1978; van Genderen 1985; Lamers et al. 1998; van Leeuwen et al. 1998). The position of the sample stars as derived by Waelkens et al. (1998) is indicated by asterisks (arrows indicate a lower limit). The new position derived in this study is marked by circles (filled - group I; grey - group II; open - group III). The dotted lines represent the ZAMS (four initial ZAMS masses - in $M_{\odot}$ - are indicated) and TAMS. Theoretical instability domains for the $\beta$ Cep (thick solid line) and the SPB stars (dashed lines) for main-sequence models are shown (Pamyatnykh 1999), together with the instability domains for post-TAMS models with $\ell=1$ (grey dotted) and $\ell=2$ (black dotted) g-modes computed by Saio et al. (2006).

$\beta$ Cep and SPB stars, derived from main sequence (pre-TAMS) models (Pamyatnykh 1999), and post-TAMS model predictions for $\ell=1$ and 2 for B stars up to $20 M_{\odot}$ (Saio et al. 2006). The former strips do not encompass our targets at first sight, whereas the latter do, at least for all stars hotter than $15000 \mathrm{~K}$. A far more appropriate comparison between the position of these strips and the sample stars is achieved from a $\left(\log T_{\text {eff }}, \log g\right)$ plot. Indeed, the position of the targets in such a diagram is much more reliable because it is free from uncertainties on the radii and quantities derived thereof. Such a plot is provided in Fig. 10. It encapsulates the major result of our study. We see that all group I stars fall very close to the higher-gravity limit of the predicted 


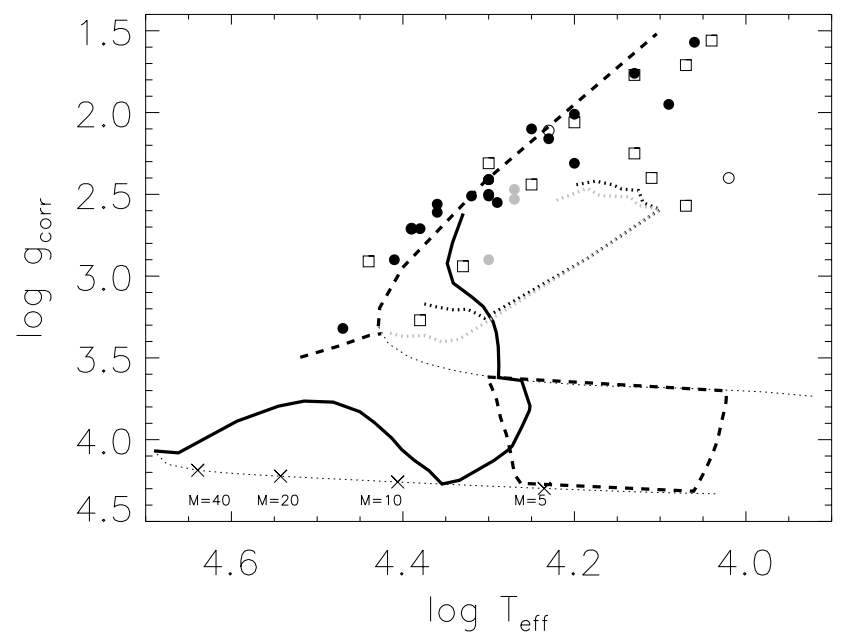

Fig. 10. Position of the sample stars and of the low-order $\mathrm{p}$-mode $(\beta \mathrm{Cep}$ - thick solid line) and high-order g-mode (SPB-type - thick dashed line) instability domains in the $\log T_{\text {eff }}-\log g$ diagram (Pamyatnykh 1999) for main sequence stars. Post-TAMS model predictions for $\ell=1$ (grey dotted) and $\ell=2$ (black dotted) g-modes are shown for B stars with masses up to $20 M_{\odot}$ (Saio et al. 2006). A few initial ZAMS masses are given in units of $M_{\odot}$. Circles have the same meaning as in Fig. 9 . Squares represent the group of comparison stars (group IV).

pre-TAMS instability strip and within the post-TAMS instability strip predictions of gravity modes in evolved stars. This implies that the observed periodic variability is indeed compatible with non-radial gravity oscillations excited by the opacity mechanism. The photometric period(s) found for our sample stars, ranging from one to a few weeks, and the high percentage of multiperiodic stars, fully support this interpretation.

The position of the comparison stars in this $\left(\log T_{\text {eff }}, \log g\right.$ ) diagram is also interesting. In Sect. 3 we argued that five among these so-called non-periodically variable supergiants, show amplitudes and periods comparable to the ones of the sample stars. We see that these five objects also lie at the same higher-gravity limit of the instability strip. From the remaining seven objects, with much lower amplitude variability, there are three that have very high (projected) rotational velocities, which result in lower amplitudes, (De Cat 2002), namely HD 64760, HD 157246, and HD 165024. Together with HD 149038, which nicely falls within the SPB instability domain, we suggest these nine targets to be newly discovered oscillators in gravity modes, unlike the remaining three objects HD 46769, HD 125288, and HD 86440, which lie, even when taking into account the error bar in $T_{\text {eff }}$, completely outside the predicted strip.

\section{Mass loss, rotation and photometric variability}

Glatzel et al. (1999) suggested the occurrence of pulsationallydriven mass loss in supergiants to be due to strange-mode instabilities on theoretical grounds. While we found evidence for non-radial gravity modes in our sample of "low-mass" stars, it is worthwile to investigate if these modes play a role in the mass loss. If this is the case, one expects a correlation between the frequency and/or amplitude of the oscillations and the mass-loss rate. In view of the normal behaviour of our sample stars in terms of the WLR relation, we can anticipate this relation to be weak, at best. Also rotation can introduce periodic variability and enhance mass loss, either alone or in combination with non-radial oscillations (Rivinius et al. 2003, and references therein).
To investigate both possible mass-loss contributors, we plot the photometric variability parameters as a function of (i) the wind density, $\log \rho_{\mathrm{S}}$, at the sonic point, (ii) the projected rotational velocity, $v \sin i$, and (iii) the ratio $\Omega$ of $v \sin i$ to the critical velocity $^{7}$ (see Fig. 11). We have chosen to consider the wind density at the sonic point (i.e., $\log \rho_{\mathrm{S}}=\log \left(\dot{M} /\left(4 \pi R_{*}^{2} c_{\mathrm{S}}\right)\right.$, with $c_{\mathrm{S}}$ the isothermal sound speed), because this is the lowermost place where mass loss is initiated and oscillations could have an effect. The Spearman rank correlation coefficients, $r$, computed for group I and II stars (indicated in each panel in Fig. 11) reveal only two mild correlations related to the mean wind density. Except for a similarly mild correlation between $\Omega$ and the peakto-peak variation, no other connection between rotation and photometric variability can be detected.

At first, a positive trend between the observed amplitude of variation and the mean wind density is visible. At the same time, we notice a tendency that higher mass-loss is present when the detected frequencies are lower, i.e., the oscillation periods are longer. In general, the oscillation periods scale as the mean density within the star so this downward trend may suggest that the role of oscillations in helping to increase the mass loss is more evident in more evolved stars. However, it concerns only weak correlations, which are not significant in a statistical sense. Moreover, the trends weaken if we add the five comparison stars with significant amplitudes.

The only seemingly "true" correlation occurs between $v \sin i$ and the mean wind density, for which we find the Spearman rank correlation coefficient to be 0.62 with a significance of $99 \%$ (Fig. 12). At first sight, one may think that this is consistent with the well-known result that rapid rotation helps in driving the mass loss. However, the correlation is not significant if we consider $\Omega$ rather than $v \sin i$. This seems to point out that the observed correlation between $v \sin i$ and $\log \rho_{\mathrm{S}}$ is a selection effect, in the sense that we mostly have very slow rotators in our sample. Indeed, a clear correlation between rotation and the mean wind density is only expected for objects rotating faster than $\sim 70 \%$ of their critical velocity, which is not at all the case for our sample stars.

Prinja \& Howarth (1986) investigated the variability in UV P Cygni profiles of early-type stars and concluded that variability in $\dot{M}$ occurs at the $10 \%$ level, on time scales of a day or longer. Changes of a factor of two or larger were never observed. Only last year, Markova et al. (2005) investigated the wind variability in $15 \mathrm{O}$ type supergiants, using $\mathrm{H} \alpha$ as a signature. They found variations of the order of $4 \%$ with respect to the mean value of $\dot{M}$ for stars with strong winds and of $\pm 16 \%$ for stars with weak winds. The ratio of maximum to minimum mass-loss rate over the time interval for their sample ranges from 1.08 to 1.47 (with a mean ratio of 1.22), with a tendency for weaker winds to show larger changes. Both numbers are fully in agreement with Prinja \& Howarth (1986). For our sample supergiants, we find that the ratio of maximum to minimum mass-loss rate ${ }^{8}$ ranges from 1.05 up to 1.88 , with a mean ratio for the whole sample of 1.31 , which is still in agreement with Prinja \& Howarth (1986), in the sense that these numbers do not exceed a factor two. On the other hand, the maximum relative differences amount to $60 \%$, with an average of $22 \%$ (without distinction between weak or strong winds), which is somewhat higher than what is expected for "normal"

7 Calculated here in the spherical approximation, since most of our objects do not rotate too fast.

8 But remember that we have only two observations at our disposal, separated by about one year. 


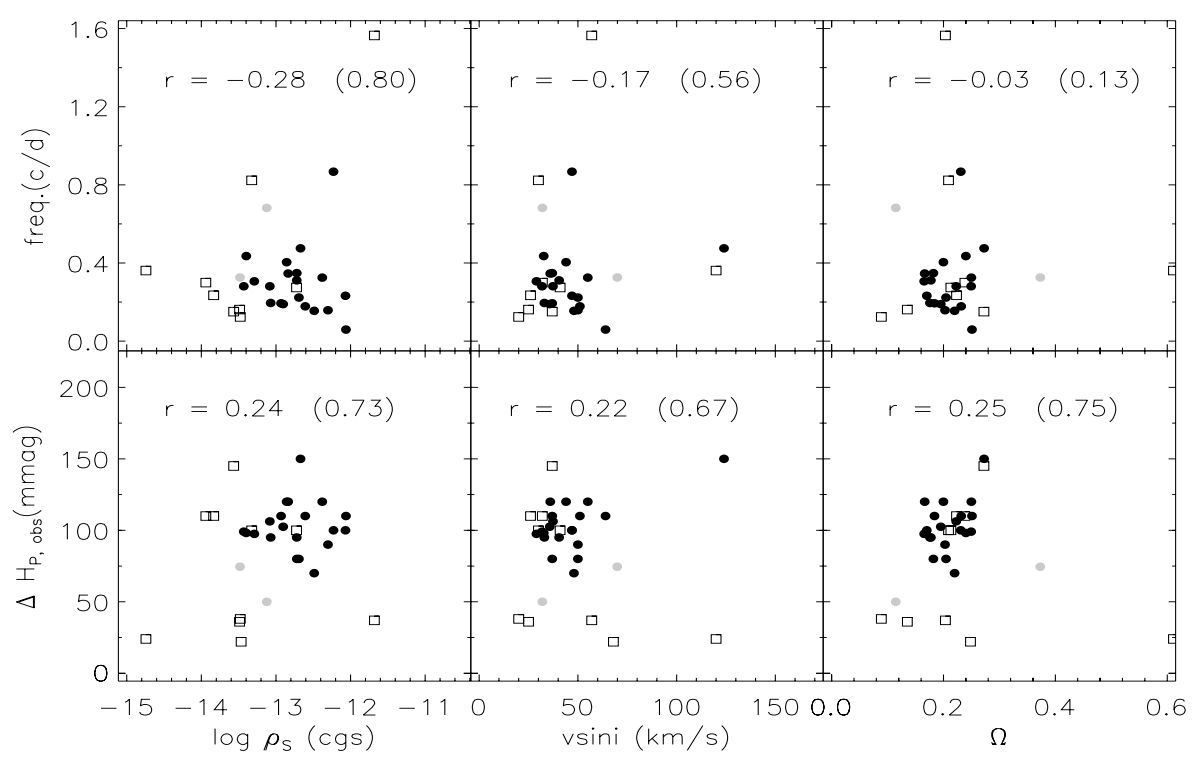

Fig. 11. Frequency and peak-to-peak variation as a function of the mean wind density, $\log \rho_{\mathrm{S}}$, at the sonic point, $v \sin i$ and $\Omega=v \sin i / v_{\text {crit }}$. Symbols have the same meaning as in Fig. 10. Spearman rank correlation coefficients, $r$, are calculated for the periodically variable B supergiants of group I and II joined. The two-sided significance is given between braces.

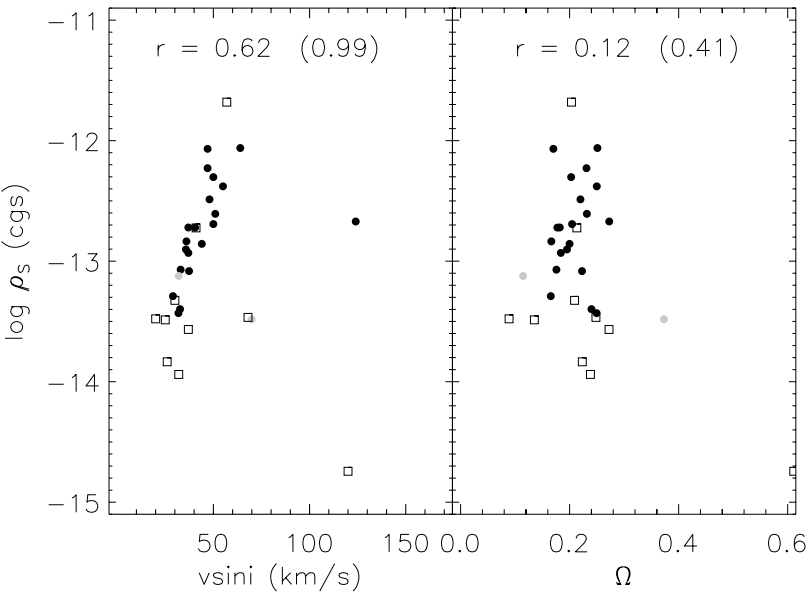

Fig. 12. Mean wind density, $\log \rho_{\mathrm{S}}$, as a function of $v \sin i$ and $\Omega$. Spearman rank correlation coefficients, $r$, for the periodically variable B supergiants are indicated (interpretation: see Fig. 11). Symbols have the same meaning as in Fig. 10.

O supergiants. This might support the idea of a connection between the mass loss and the variability in these stars.

At present we cannot conclude definitively to have found evidence for mass-loss enhancement through oscillations. We merely find an indication that higher photometric amplitudes seem to be accompanied by a higher wind density and a higher relative change in the mass-loss rate. The validity of these suggested connections would merit from further study through a more refined time-resolved analysis of both the oscillation and wind properties of a few selected targets.

\section{Discussion and summary}

We derived stellar and wind parameters of 40 B-type supergiants using the NLTE atmosphere code FASTWIND and highresolution, high signal-to-noise spectroscopic data of selected $\mathrm{H}$, $\mathrm{He}$, and $\mathrm{Si}$ lines. For the majority of the objects (excluding group III), these parameters are accurate within the discussed assumptions, and reliable error bars have been estimated as well. The primary aim of our study was to investigate if the origin of the variability found in HIPPARCOS data of 28 of these stars could be gravity-mode oscillations excited by the opacity mechanism that is also at work in main sequence B stars. To assess this suggestion made by Waelkens et al. (1998), we needed to achieve accurate values of the effective temperature and gravity of the sample stars to compare them with those of stellar models for which theoretical computations predict oscillations. The conclusion is clear cut: all the sample stars for which we could derive reliable stellar parameters have $T_{\text {eff }}$ and $\log g$ values consistent with those of stellar models in which gravity modes are predicted. They are all situated at the high $\log g$ limit of the instability strip of gravity modes in evolved stars, covering the entire range in spectral type B. Due to the lack of a clear correlation between the observed photometric variability and the stellar rotation, and in view of the large fraction of stars with multiperiodic behaviour, we suggest our sample stars to be opacity-driven non-radial oscillators.

Our study involved a sample of twelve comparison B supergiants that were not selected to be periodically variable by the HIPPARCOS team. Nevertheless, we found that nine of these behave similarly to our original sample stars and we suggest them to be new $\alpha$ Cyg variables with gravity modes as well. We thus end up with a sample of 37 non-radial pulsators in the upper part of the HR diagram. The occurrence of opacity-driven oscillations in that area was recently proven to be correct for the B2 Ib/II star HD 163899 from a 37-day ultra-precise MOST space-based photometric lightcurve (Saio et al. 2006). This star was found to oscillate in 48 frequencies with amplitudes below 4 mmag. Our findings and those by Saio et al. (2006) open up the area of asteroseismology to the upper-part of the HR diagram and lead to excellent prospects for fine-tuning of evolutionary models from the ZAMS towards the supernova stage from the seismic sounding of B supergiant interiors. To achieve this, one needs to obtain long-term high-precision time series in photometry and spectroscopy to achieve mode identification, and derive accurate stellar parameters in the way achieved in this paper. 
We find marginal evidence for a connection between the photometric amplitudes and the wind density, in the sense that the oscillations may help the line driving at the base of the wind. Firm conclusions in this direction require further detailed study of a few sample stars though. Since our sample stars display similar WLRs as found for "normal" B-supergiants (if there is something like "normal" at all), the enhancement of the mass loss cannot be very strong. Interestingly, however, we also found one (important) difference compared to previous evidence. Whereas the results from Kudritzki et al. (1999) strongly implied that all mid B-type supergiants display a WLR that lies significantly below the theoretically predicted one, our findings show that there is no such unique (and problematic) correlation. Part of our sample objects show this dilemma indeed (in particular those with $\log L / L_{\odot}>5$ ), whereas lower luminosity objects were found to be rather consistent with theory. In this investigation we also derived, for the first time, wind momenta of late-type B-supergiants, which follow the trend of A-supergiants surprisingly well.

While trying to achieve a high-precision estimate of the stellar and wind parameters through line-profile fitting, we came across several interesting features and results. The most important one is that we found ways to achieve good estimates for the parameters, despite the limited number of available lines, thanks to the high predictive power for different parameter estimates of each of the available lines. We reached this conclusion because we could compare our analyses with similar ones based on more spectral lines available in the literature. This also led to a new effective temperature calibration in good agreement with previous ones. We used this calibration to provide a practical recipe to compute $T_{\text {eff }}$ as a function of spectral type. We are currently automating the fitting procedure and will apply our methodology to a large sample of early-type stars, comprising all massive stars in the archive with ground-based preparatory observations for the COROT space mission (Solano et al. 2005), for which we have full spectra at our disposal.

Acknowledgements. We thank P. Crowther for providing us with the CTIO/JKT spectra for the stars we had in common, and A. A. Pamyatnykh for providing us with the theoretical instability domains B stars. K.L. and C.A. are supported by the Research Council of the Catholic University of Leuven under grant GOA/2003/04. We thank T. Vanneste, B. Nicolai, and L. Eylenbosch for their contribution to this study in the framework of their Master Theses and Drs. P. De Cat, L. Decin, and J. De Ridder for their contribution to the data gathering.

\section{References}

Abt, H. A., \& Cardona, O. 1984, ApJ, 285, 190

Aerts, C. 2000a, in The Third MONS Workshop: Science Preparation and Target Selection, ed. T. Teixeira, \& T. Bedding, 131

Aerts, C. 2000b, A\&A, 361, 245

Aerts, C., \& De Cat, P. 2003, Space Sci. Rev., 105, 453

Aerts, C., De Cat, P., Peeters, E., et al. 1999, A\&A, 343, 872

Asplund, M., Grevesse, N., \& Sauval, A. J. 2005, in Cosmic Abundances as

Records of Stellar Evolution and Nucleosynthesis, ed. T. G. Barnes, III \& F. N. Bash, ASP Conf. Ser., 336, 25

Balona, L. A., \& Dziembowski, W. A. 1999, MNRAS, 309, 221

Belikov, A. N., Kharchenko, N. V., Piskunov, A. E., \& Schilbach, E. 1999, A\&AS, 134, 525

Bianchi, L., Lamers, H. J. G. L. M., Hutchings, J. B., et al. 1994, A\&A, 292, 213

Bosch, G. L., Morrell, N. I., \& Niemelä, V. S. 1999, Rev. Mex. Astron. Astrofis., 35,85

Bresolin, F., Kudritzki, R.-P., Lennon, D. J., et al. 2002, ApJ, 580, 213

Burki, G. 1978, A\&A, 65, 357

Burki, G., Maeder, A., \& Rufener, F. 1978, A\&A, 65, 363

Crowther, P. A., Lennon, D. J., \& Walborn, N. R. 2006, A\&A, 446, 279

Daflon, S., \& Cunha, K. 2004, ApJ, 617, 1115
De Cat, P. 2002, in ASP Conf. Ser., 196

Eggen, O. J. 1986, AJ, 92, 1074

ESA 1997, The Hipparcos and Tycho Catalogues, Hipparcos Variability Annex: Periodic and Unsolved Variables - Spectral Types Volume 11 (ESA)

Evans, C. J., Lennon, D. J., Walborn, N. R., Trundle, C., \& Rix, S. A. 2004, PASP, 116, 909

Evans, C. J., Smartt, S. J., Lee, J.-K., et al. 2005, A\&A, 437, 467

Fabbian, D., Recio-Blanco, A., Gratton, R. G., \& Piotto, G. 2005, A\&A, 434, 235

Fullerton, A. W., Massa, D. L., \& Prinja, R. K. 2006, ApJ, 637, 1025 Georgelin, Y. M., Russeil, D., Marcelin, M., et al. 1996, A\&AS, 120, 41

Gies, D. R., \& Lambert, D. L. 1992, ApJ, 387, 673

Glatzel, W., \& Kiriakidis, M. 1993, MNRAS, 262, 85

Glatzel, W., Kiriakidis, M., Chernigovskij, S., \& Fricke, K. J. 1999, MNRAS, 303,116

Gray, D. F. 1973, ApJ, 184, 461

Gray, D. F. 1978, Sol. Phys., 59, 193

Grevesse, N., \& Sauval, A. J. 1998, Space Sci. Rev., 85, 161

Gummersbach, C. A., Kaufer, A., Schaefer, D. R., Szeifert, T., \& Wolf, B. 1998, A\&A, 338, 881

Herrero, A., Puls, J., \& Najarro, F. 2002, A\&A, 396, 949

Hillenbrand, L. A., Massey, P., Strom, S. E., \& Merrill, K. M. 1993, AJ, 106, 1906

Hillier, D. J., \& Miller, D. L. 1998, ApJ, 496, 407

Howarth, I. D., Townsend, R. H. D., Clayton, M. J., et al. 1998, MNRAS, 296, 949

Hubeny, I., \& Lanz, T. 2000, BAAS, 32, 1531

Kaufer, A., Stahl, O., Wolf, B., et al. 1997, A\&A, 320, 273

Kaufer, A., Prinja, R. K., \& Stahl, O. 2002, A\&A, 382, 1032

Kaufer, A., Stahl, O., Prinja, R. K., \& Witherick, D. 2006, A\&A, 447, 325

Kestens, E. 1998, Master Thesis, K. U. Leuven

Kilian, J. 1992, A\&A, 262, 171

Kilian, J., Montenbruck, O., \& Nissen, P. E. 1994, A\&A, 284, 437

Kubát, J., Puls, J., \& Pauldrach, A. W. A. 1999, A\&A, 341, 587

Kudritzki, R., \& Puls, J. 2000, ARA\&A, 38, 613

Kudritzki, R. P., Puls, J., Lennon, D. J., et al. 1999, A\&A, 350, 970

Lamers, H. J. G. L. M., Snow, T. P., \& Lindholm, D. M. 1995, ApJ, 455, 269

Lamers, H. J. G. L. M., Bastiaanse, M. V., Aerts, C., \& Spoon, H. W. W. 1998, A\&A, 335, 605

Lamers, H. J. G. L. M., Haser, S., de Koter, A., \& Leitherer, C. 1999, ApJ, 516, 872

Lennon, D. J., Dufton, P. L., \& Fitzsimmons, A. 1993, A\&AS, 97, 559

Lovy, D., Maeder, A., Noels, A., \& Gabriel, M. 1984, A\&A, 133, 307

Lyubimkov, L. S., Rostopchin, S. I., \& Lambert, D. L. 2004, MNRAS, 351, 745

Maíz-Apellániz, J., Walborn, N. R., Galué, H. Á., \& Wei, L. H. 2004, ApJS, 151, 103

Marchenko, S. V., Moffat, A. F. J., van der Hucht, K. A., et al. 1998, A\&A, 331, 1022

Markova, N., Puls, J., Repolust, T., \& Markov, H. 2004, A\&A, 413, 693

Markova, N., Puls, J., Scuderi, S., \& Markov, H. 2005, A\&A, 440, 1133

Martins, F., Schaerer, D., \& Hillier, D. J. 2005, A\&A, 436, 1049

Massey, P., Bresolin, F., Kudritzki, R. P., Puls, J., \& Pauldrach, A. W. A. 2004, ApJ, 608, 1001

Massey, P., Puls, J., Pauldrach, A. W. A., et al. 2005, ApJ, 627, 477

Mathias, P., Aerts, C., Briquet, M., et al. 2001, A\&A, 379, 905

McErlean, N. D., Lennon, D. J., \& Dufton, P. L. 1998, A\&A, 329, 613

McErlean, N. D., Lennon, D. J., \& Dufton, P. L. 1999, A\&A, 349, 553

Michaud, G., Vauclair, G., \& Vauclair, S. 1983, ApJ, 267, 256

Mokiem, M. R., de Koter, A., Puls, J., et al. 2005, A\&A, 441, 711

Mokiem, M. R., de Koter, A., Evans, C. J., et al. 2006, A\&A, 456, 1131

Morel, T., Marchenko, S. V., Pati, A. K., et al. 2004, MNRAS, 351, 552

Najarro, F., Hillier, D. J., Puls, J., Lanz, T., \& Martins, F. 2006, A\&A, 456, 659

Pamyatnykh, A. A. 1999, Acta Astron., 49, 119

Pauldrach, A. W. A., Hoffmann, T. L., \& Lennon, M. 2001, A\&A, 375, 161

Paunzen, E., \& Maitzen, H. M. 1998, A\&AS, 133, 1

Piters, A. J. M., Groot, P. J., \& van Paradijs, J. 1996, A\&AS, 118, 529

Prinja, R. K., \& Howarth, I. D. 1986, ApJS, 61, 357

Prinja, R. K., \& Massa, D. L. 1998, in Properties of Hot Luminous Stars, ed. I. Howarth, ASP Conf. Ser., 131, 218

Prinja, R. K., Massa, D., Fullerton, A. W., Howarth, I. D., \& Pontefract, M. 1997, A\&A, 318, 157

Prinja, R. K., Massa, D., \& Fullerton, A. W. 2002, A\&A, 388, 587

Przybilla, N., Butler, K., Becker, S. R., \& Kudritzki, R. P. 2006, A\&A, 445, 1099

Puls, J., Kudritzki, R.-P., Herrero, A., et al. 1996, A\&A, 305, 171

Puls, J., Urbaneja, M. A., Venero, R., et al. 2005, A\&A, 435, 669

Puls, J., Markova, N., Scuderi, S., et al. 2006, A\&A, 454, 625

Repolust, T., Puls, J., Hanson, M. M., Kudritzki, R.-P., \& Mokiem, M. R. 2005,

A\&A, 440, 261 
Repolust, T., Puls, J., \& Herrero, A. 2004, A\&A, 415, 349

Rivinius, T., Baade, D., \& Štefl, S. 2003, A\&A, 411, 229

Rolleston, W. R. J., Smartt, S. J., Dufton, P. L., \& Ryans, R. S. I. 2000, A\&A, 363,537

Ryans, R. S. I., Dufton, P. L., Rolleston, W. R. J., et al. 2002, MNRAS, 336, 577 Saio, H., Kuschnig, R., Gautschy, A., et al. 2006, ApJ, 650, 1111

Santolaya-Rey, A. E., Puls, J., \& Herrero, A. 1997, A\&A, 323, 488

Scargle, J. D. 1982, ApJ, 263, 835

Schmidt-Kaler, T. 1982, Physical Parameters of the Stars, Landolt-Börnstein: Numerical Data and Functional Relationships in Science and Technology New Series, Group VI, Volume 2b (Berlin: Springer-Verslag)

Simón-Díaz, S., Herrero, A., Esteban, C., \& Najarro, F. 2006, A\&A, 448, 351

Slowik, D. J., \& Peterson, D. M. 1995, AJ, 109, 2193

Solano, E., Catala, C., Garrido, R., et al. 2005, AJ, 129, 547

Stellingwerf, R. F. 1978, ApJ, 224, 953
Sterken, C. 1977, A\&A, 57, 361

Sterken, C. 1983, The Messenger, 33, 10

Trundle, C., Lennon, D. J., Puls, J., \& Dufton, P. L. 2004, A\&A, 417, 217

Urbaneja, M. A. 2004, Ph.D. Thesis, Instituto de Astrofisica de Canarias

Urbaneja, M. A., Herrero, A., Kudritzki, R. P., et al. 2002, A\&A, 386, 1019 van Genderen, A. M. 1985, A\&A, 151, 349

van Genderen, A. M. 2001, A\&A, 366, 508

van Genderen, A. M., Bovenschen, H., Engelsman, E. C., et al. 1989, A\&AS,

$$
79,263
$$

van Leeuwen, F., van Genderen, A. M., \& Zegelaar, I. 1998, A\&AS, 128, 117

Vink, J. S., de Koter, A., \& Lamers, H. J. G. L. M. 2000, A\&A, 362, 295

Waelkens, C., Aerts, C., Kestens, E., Grenon, M., \& Eyer, L. 1998, A\&A, 330, 215

Walborn, N. R., \& Bohlin, R. C. 1996, PASP, 108, 477

Zsargó, J., Hillier, D. J., \& Georgiev, L. N. 2006, A\&A, 447, 1093 
K. Lefever et al.: Periodically variable B supergiants, Online Material p 1

\section{Online Material}


K. Lefever et al.: Periodically variable B supergiants, Online Material p 2

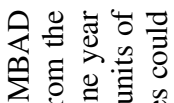

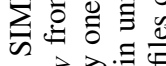

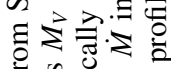

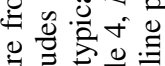

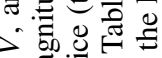

ง

정

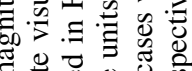

记

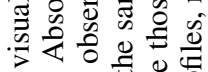

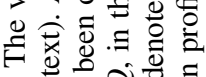

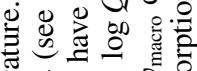

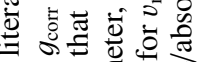

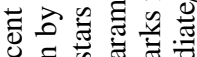

论

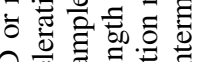

安论

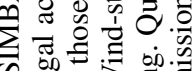

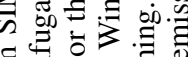

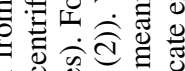

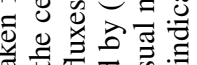

西

记

20

过

议

政的

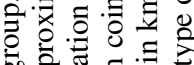

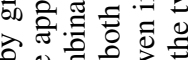

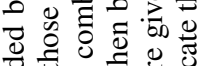

过

记

के

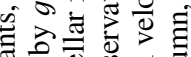

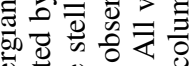

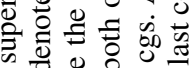

을

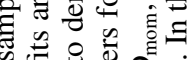

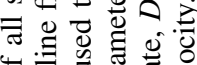

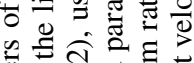

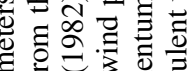

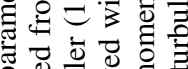

记

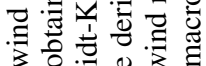

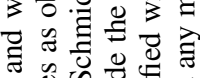

สิ

흥

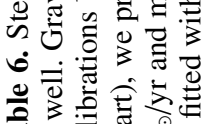

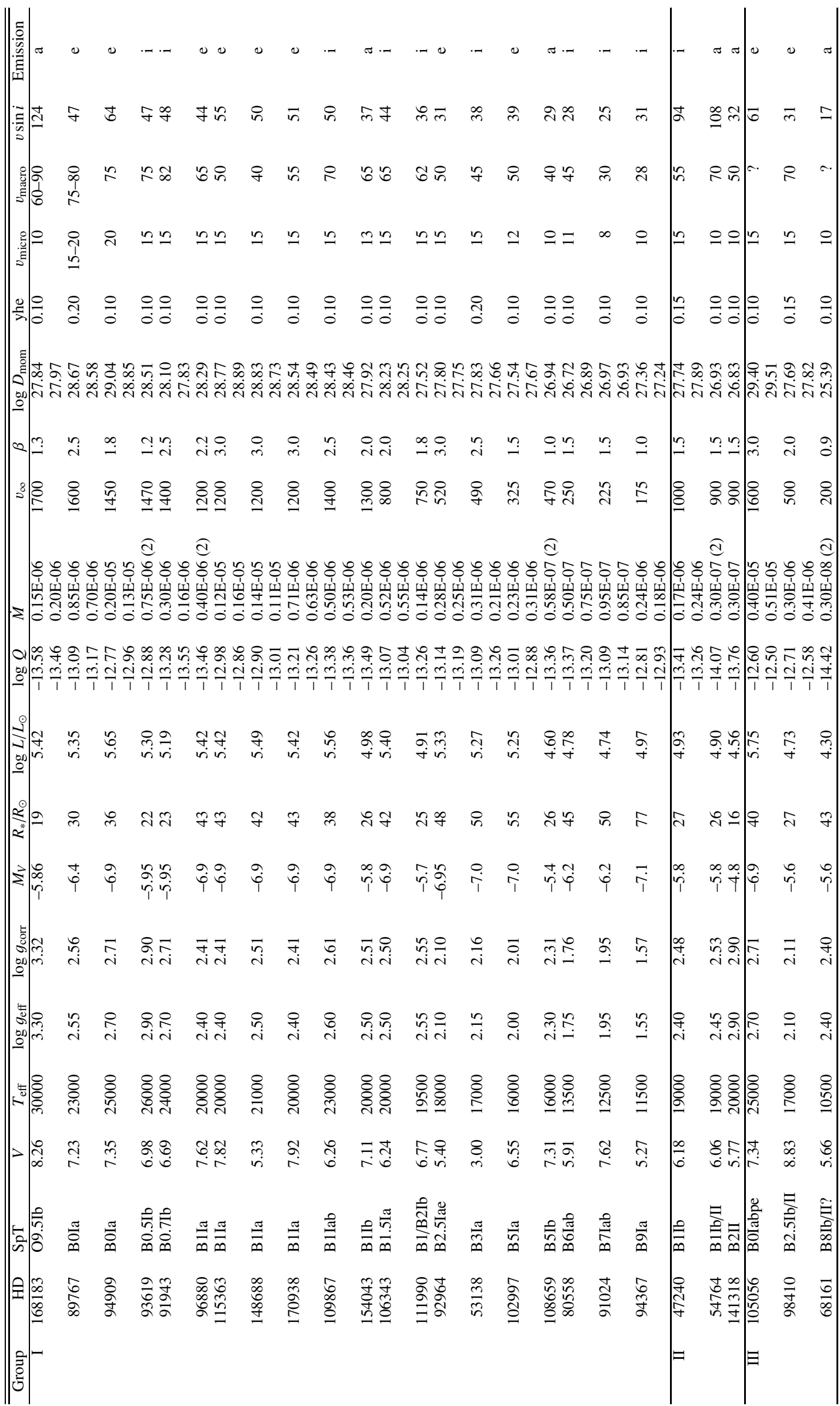


K. Lefever et al.: Periodically variable B supergiants, Online Material p 3

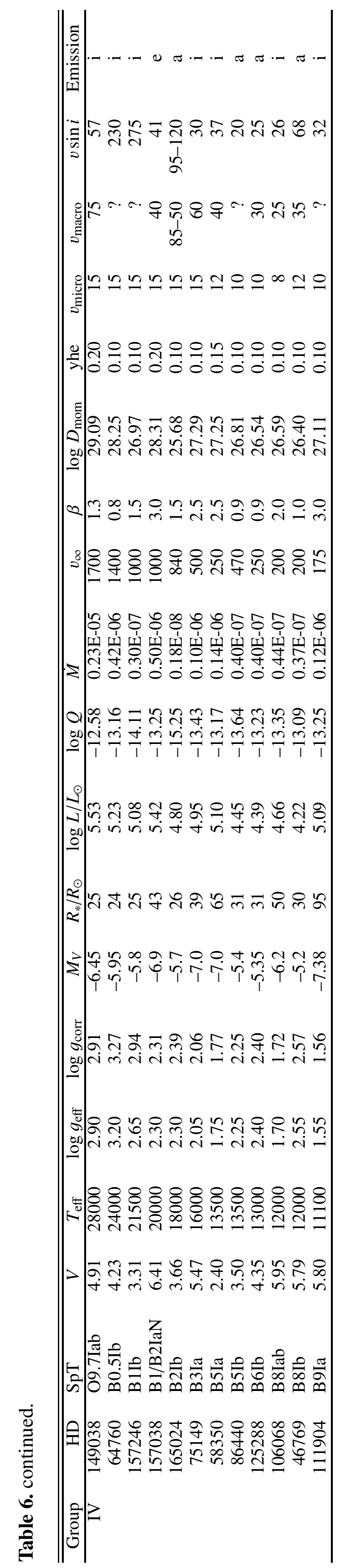


K. Lefever et al.: Periodically variable B supergiants, Online Material p 4

\section{Appendix A: Spectral line fits of all sample and comparison stars}

$\mathrm{H} \alpha 1$

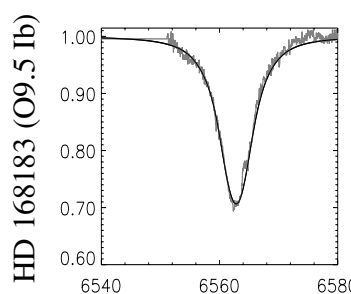

$\mathrm{H} \alpha 2$
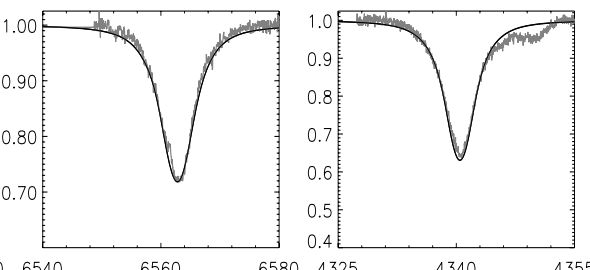

He I 4471

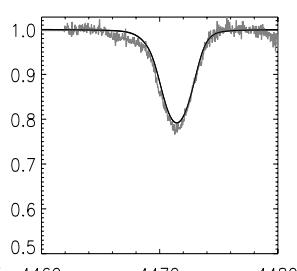

He I 6678

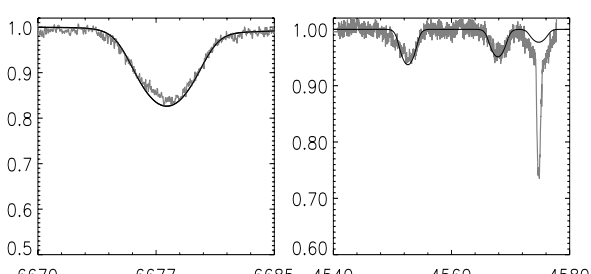

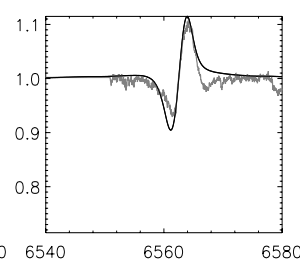
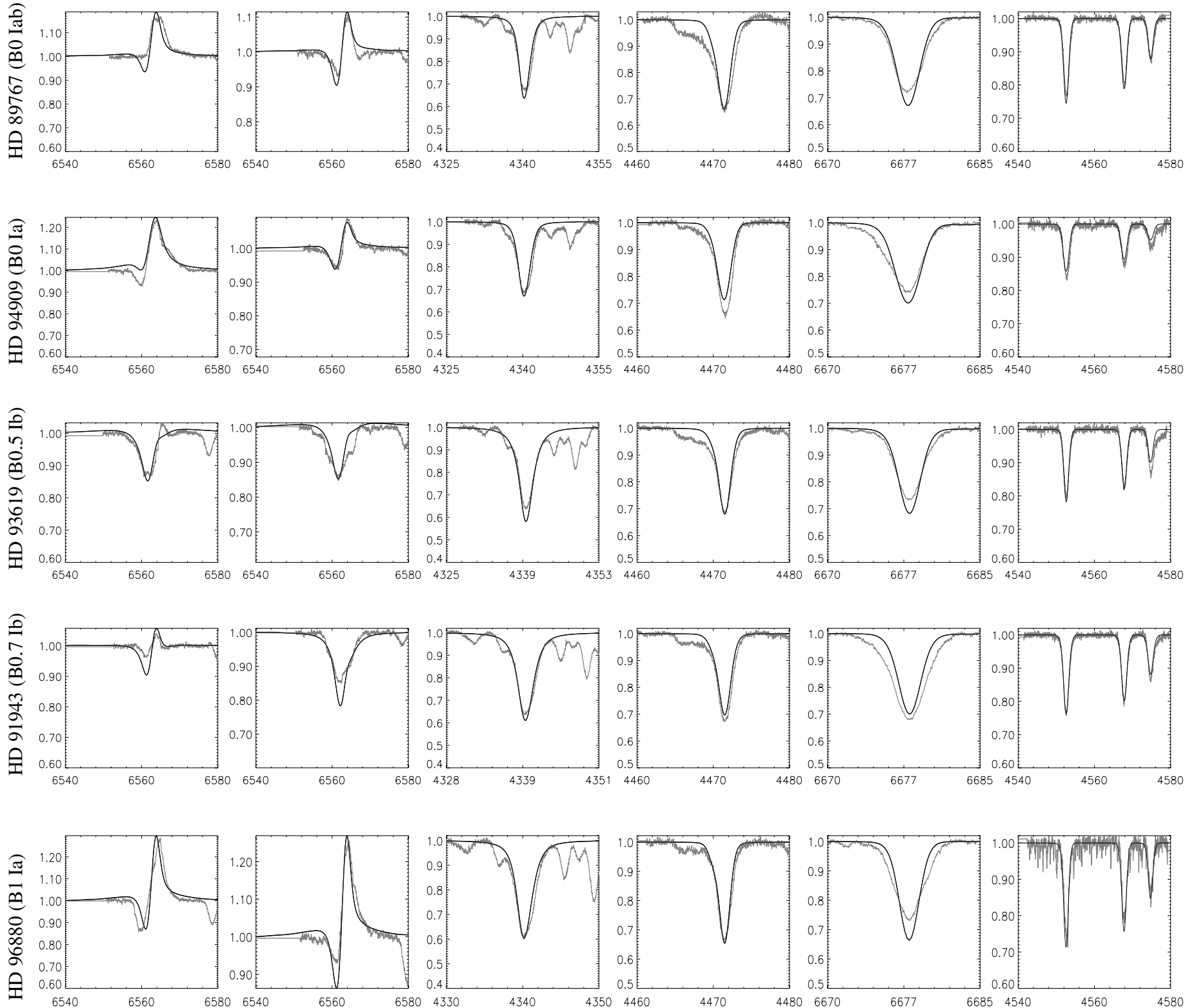

Fig. A.1. Spectral line fits for the periodically variable B-type supergiants with reliable parameters: group I. 
K. Lefever et al.: Periodically variable B supergiants, Online Material p 5

$\mathrm{H} \alpha 1$
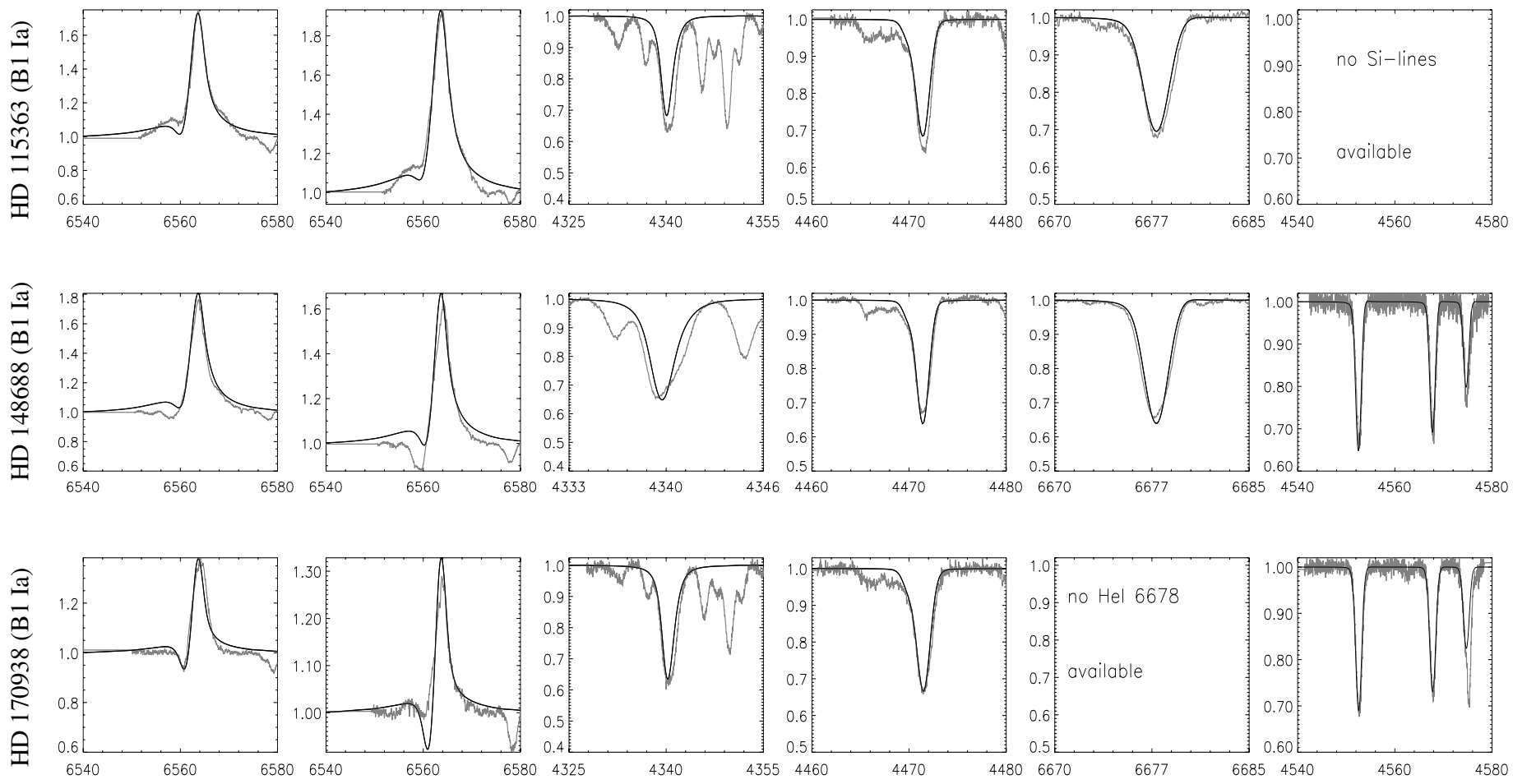

जิ
0
0
6
0
8
0
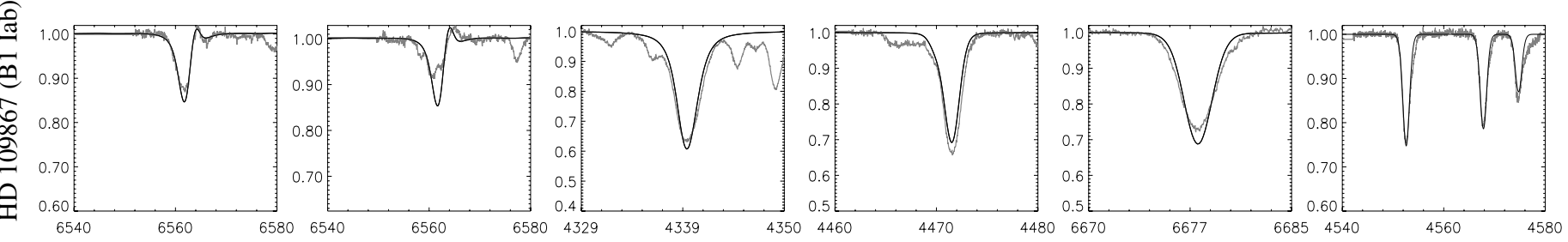

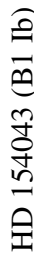
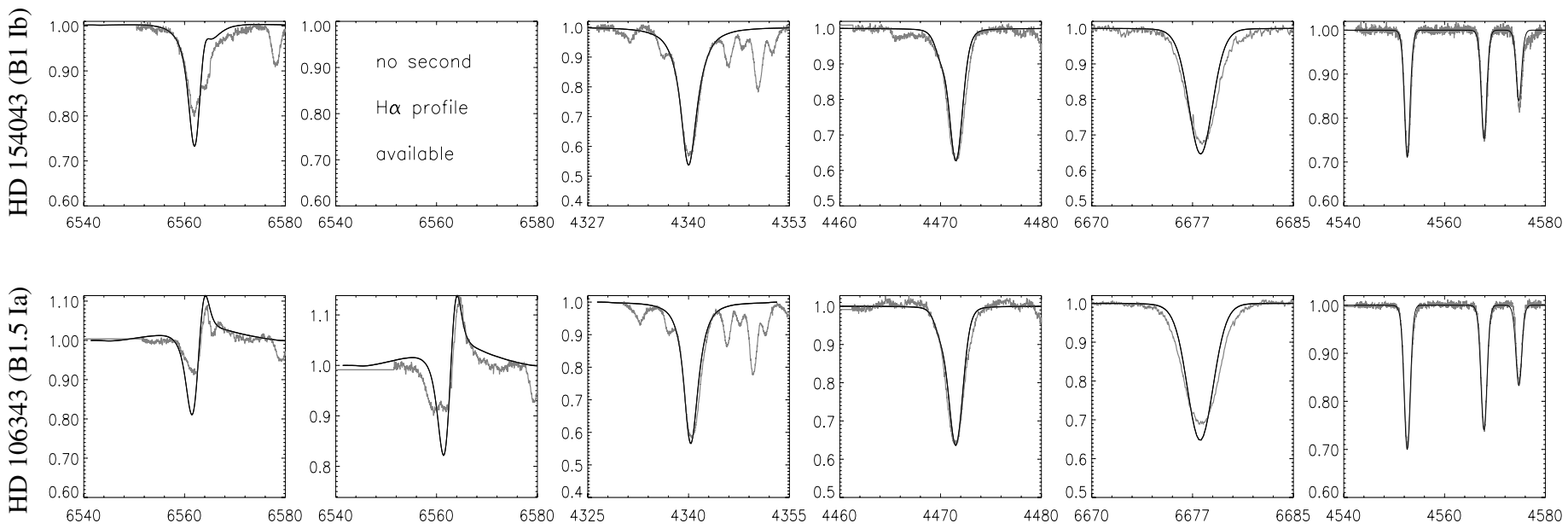

Fig. A.1. continued. 
K. Lefever et al.: Periodically variable B supergiants, Online Material p 6

$\mathrm{H} \alpha 1$
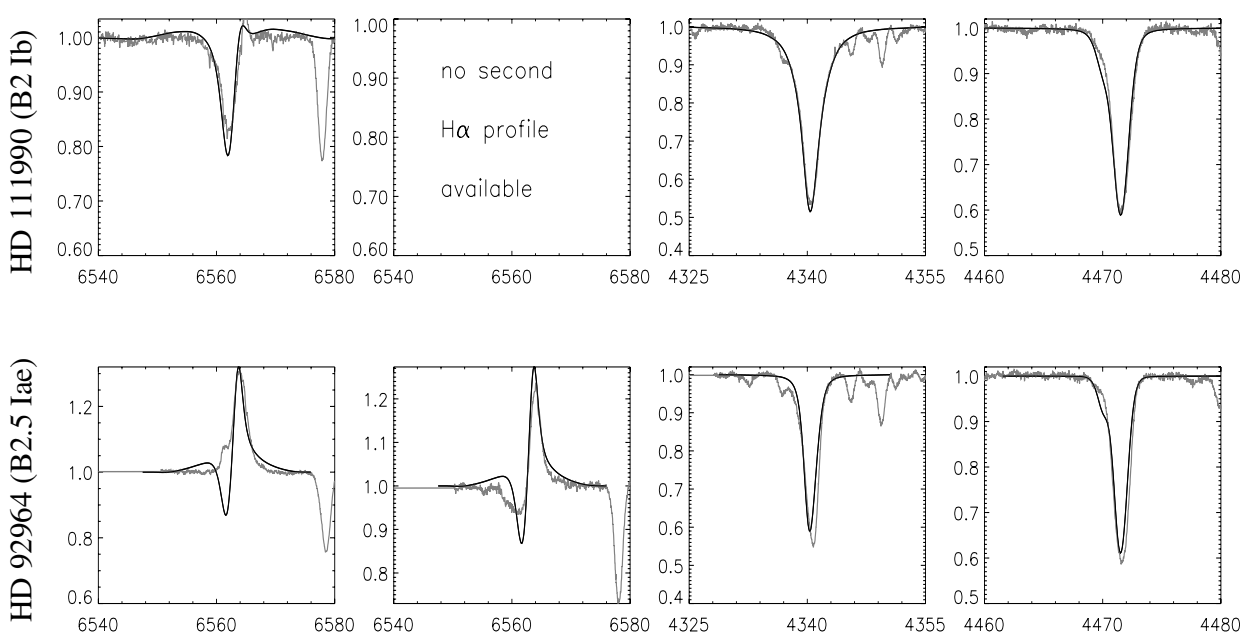

He I 6678

Si II
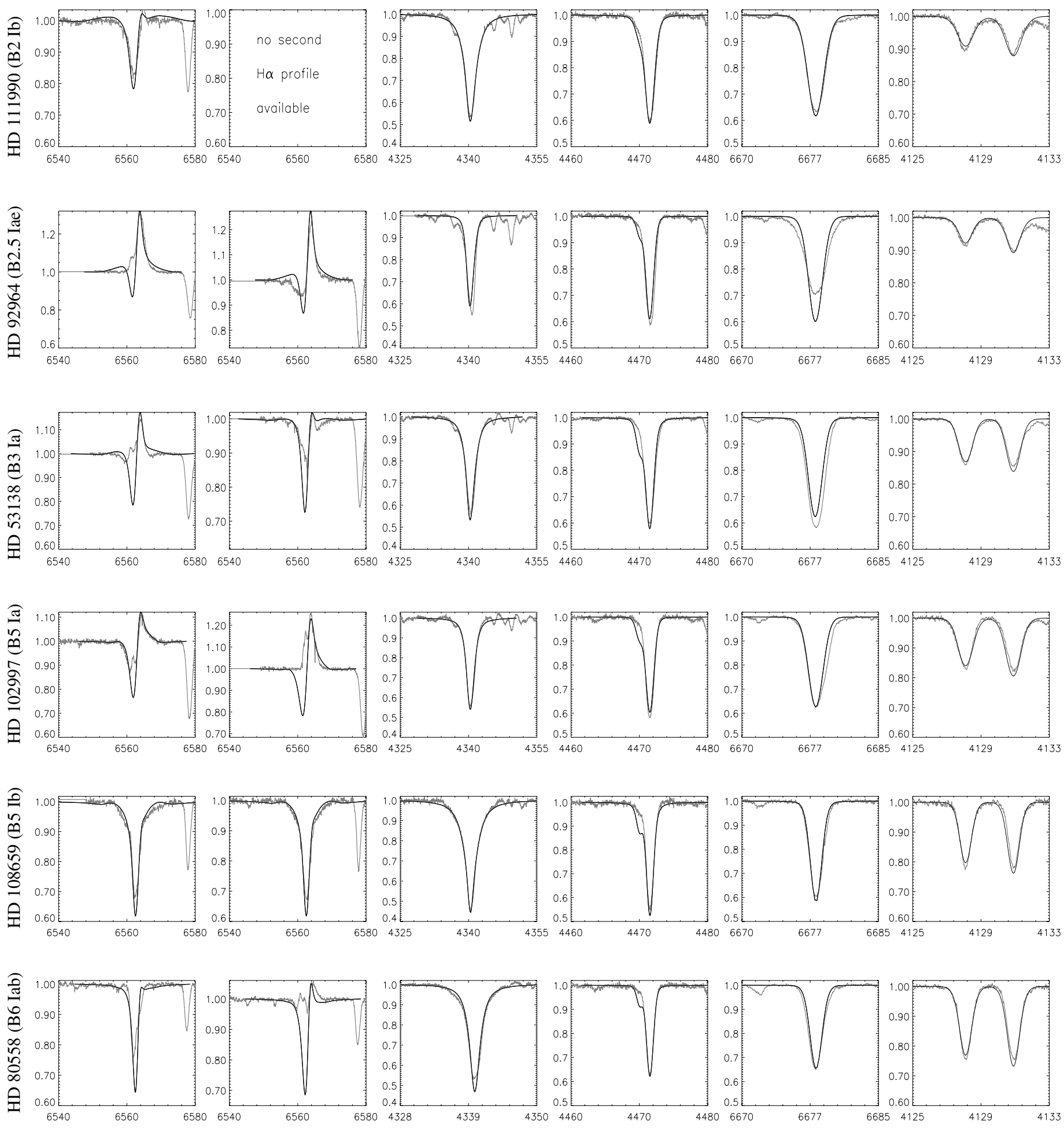

Fig. A.1. continued. 
K. Lefever et al.: Periodically variable B supergiants, Online Material $p 7$

$\mathrm{H} \alpha 1$

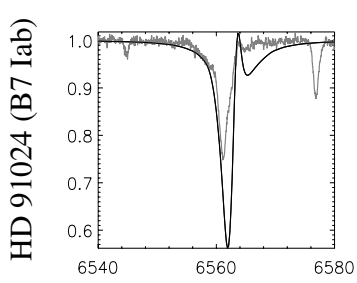

$\mathrm{H} \alpha 2$
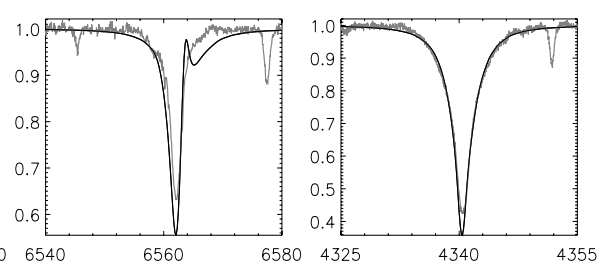

He I 4471

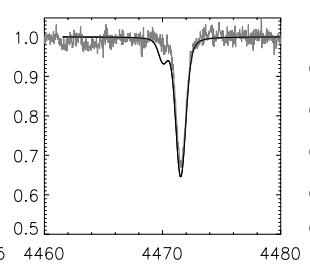

He I 6678

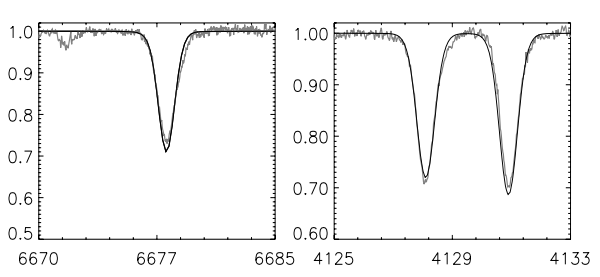

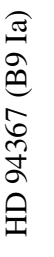
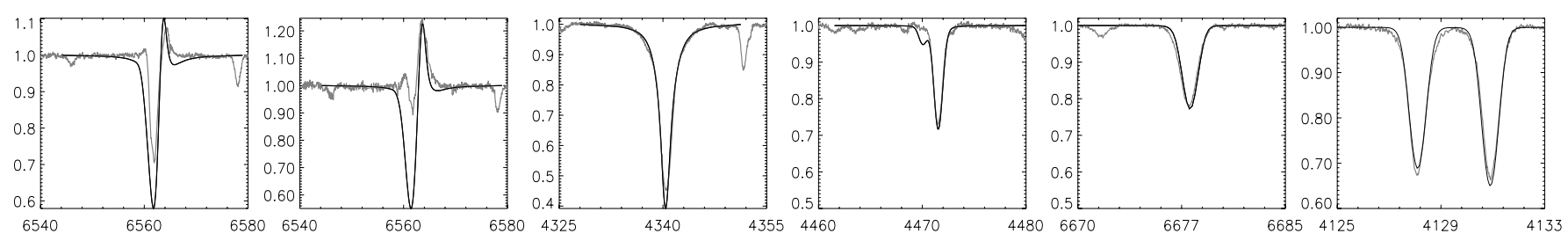

Fig. A.1. continued.

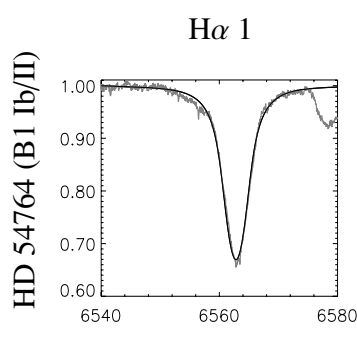

$\mathrm{H} \alpha 2$
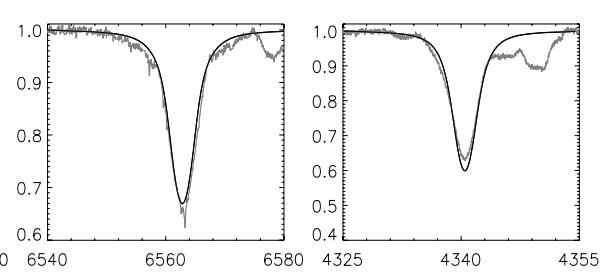

He I 4471

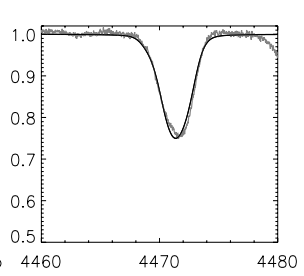

He I 6678
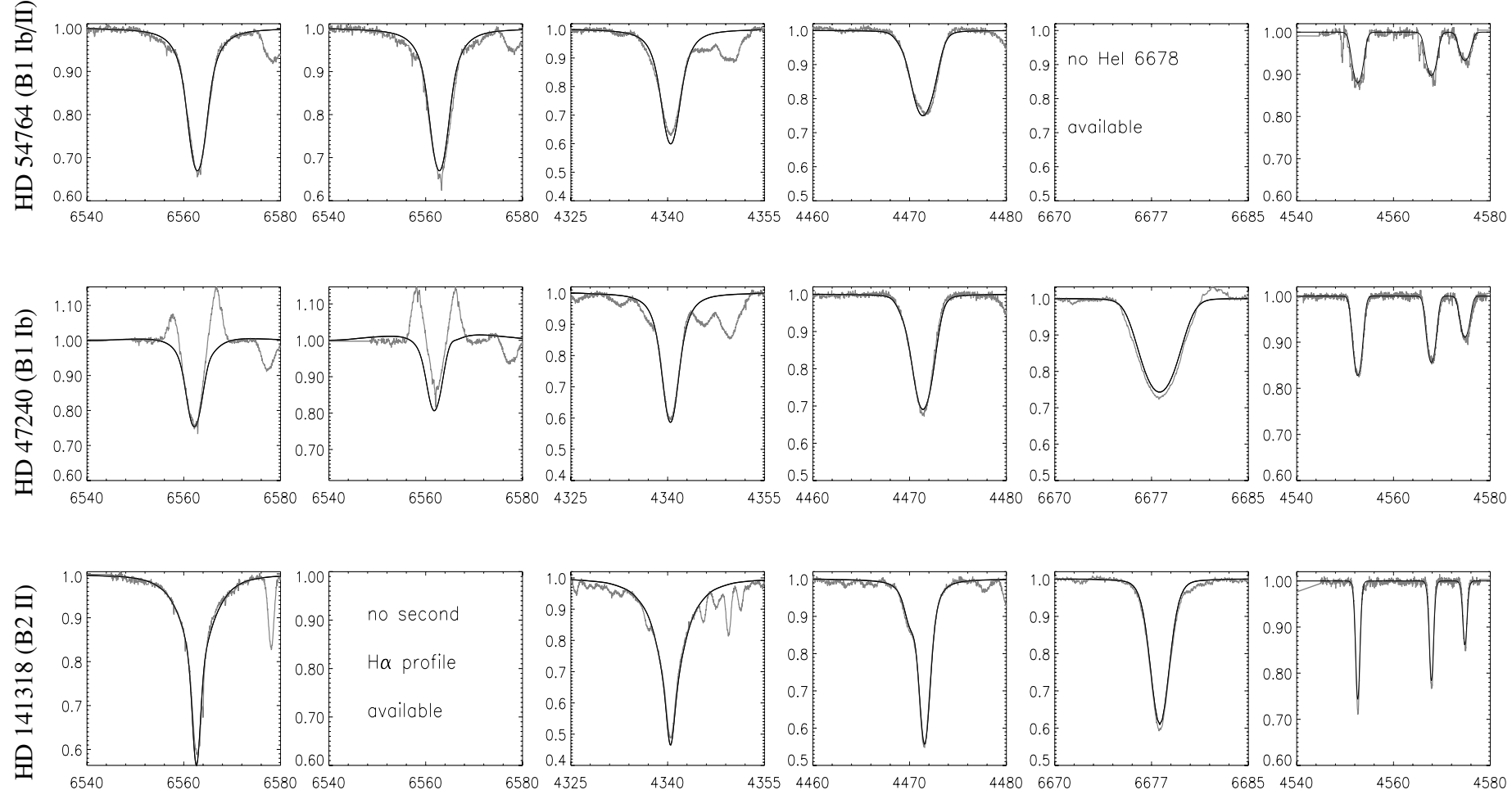

Fig. A.2. Spectral line fits for the periodically variable B-type supergiants with a potential $T_{\text {eff }}$ dichotomy: group II. Only our favoured solution is displayed (see text). 
K. Lefever et al.: Periodically variable B supergiants, Online Material $p 8$

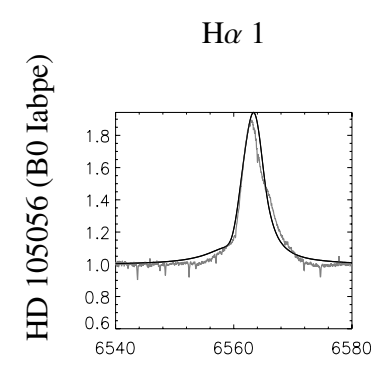

$\mathrm{H} \alpha 2$

$\mathrm{H} \gamma$

He I 4471

He I 6678

Si II/III
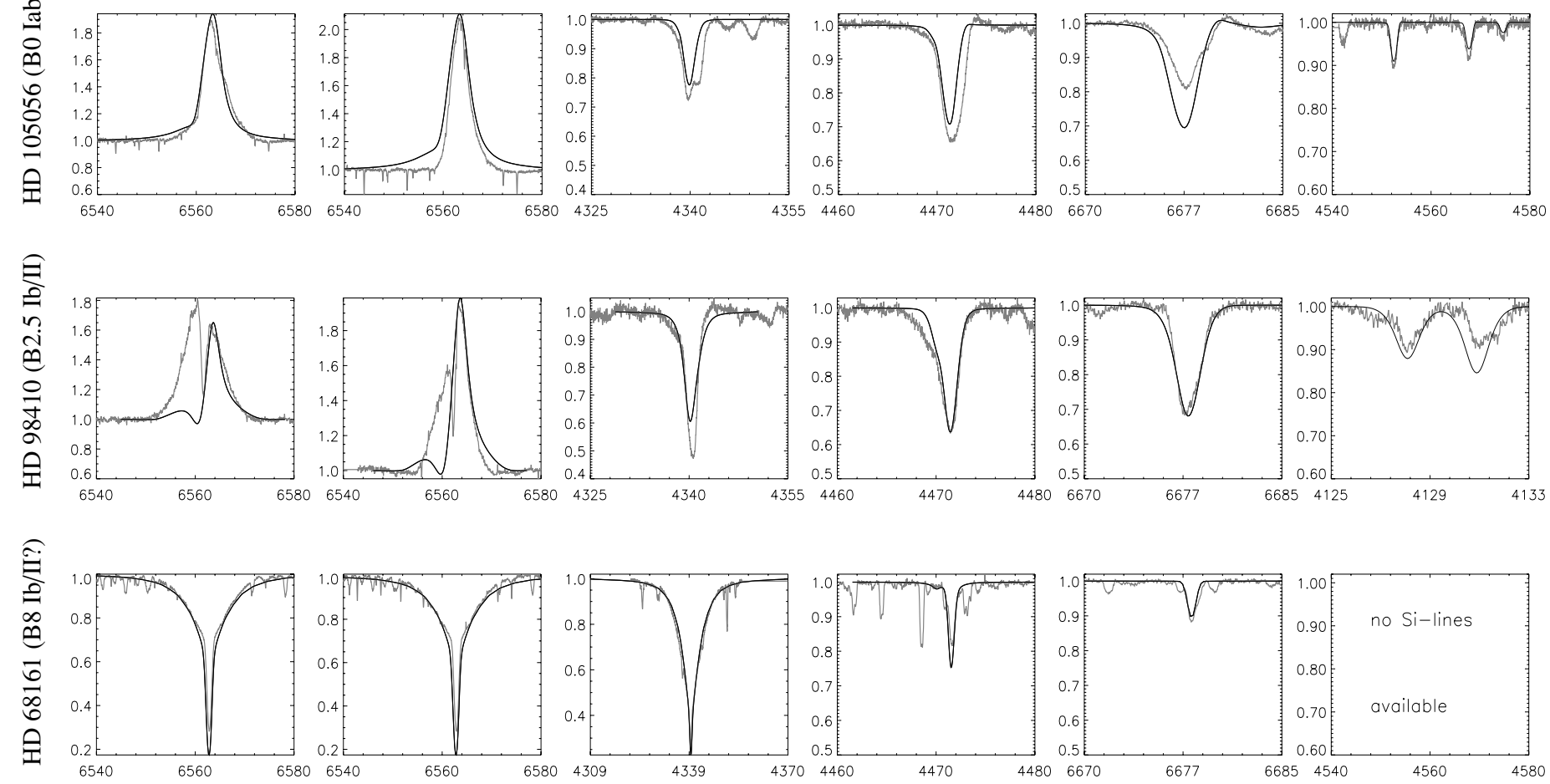

Fig. A.3. Spectral line fits for the periodically variable B-type supergiants with parameters to be considered as indicative numbers only: group III. 
K. Lefever et al.: Periodically variable B supergiants, Online Material $p 9$
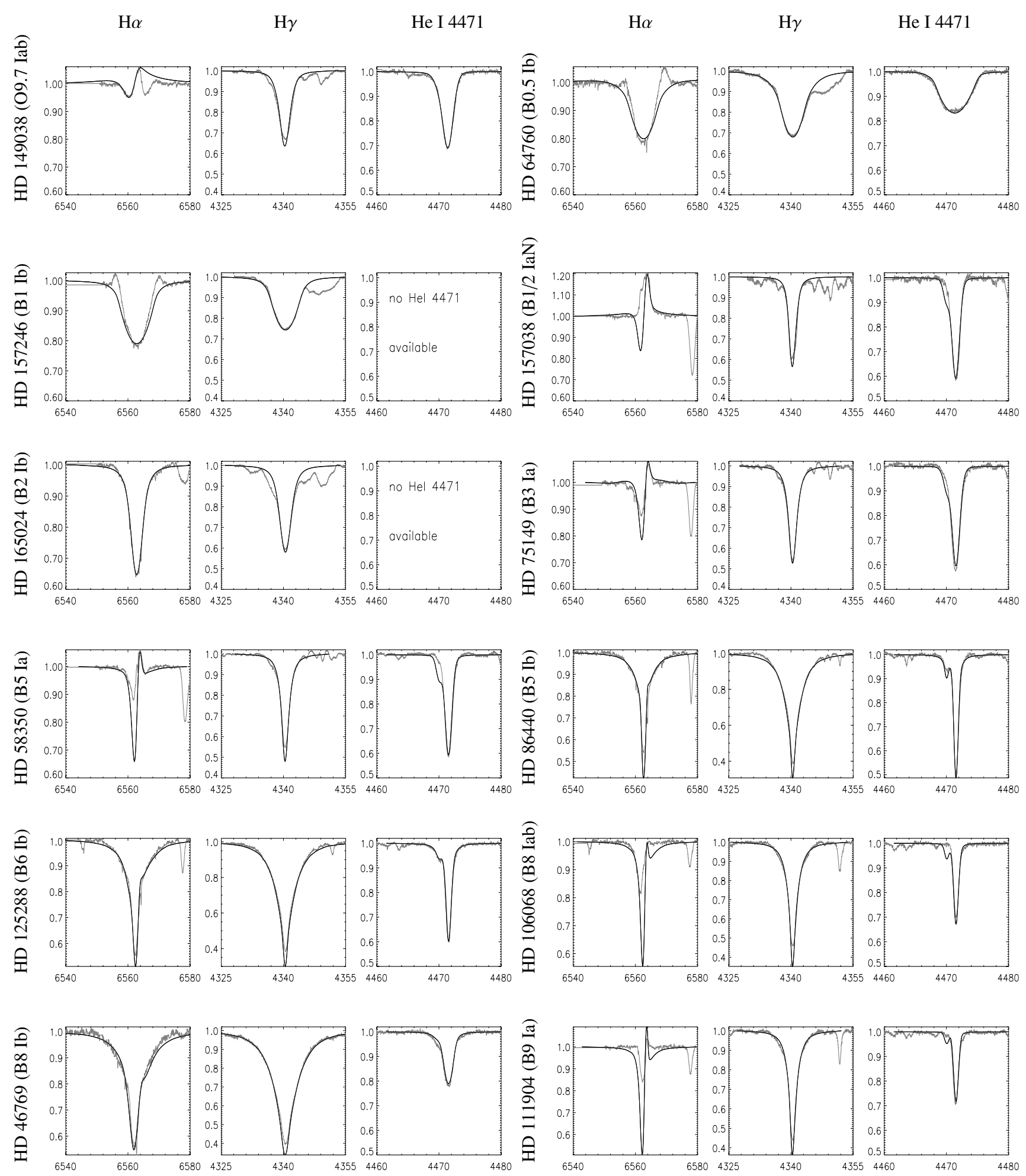

Fig. A.4. Spectral line fits for the comparison stars (previously not known to exhibit any periodic variability). No Si available. 


\section{Appendix B: Individual discussion of all sample and comparison stars}

In the following, we discuss all sample and comparison stars one by one and group per group. The order of description is based on the spectral type of each object as adopted in this paper (see discussion of individual objects below and also Sect. 7). Within each spectral type, stars are ordered following their HD number. This order refers to the order of the objects in Figs. A.1. to A.7. as well.

\section{B.1. Stars discarded from the sample}

We have good reasons to remove HD 51110 (B9 Ib/II) from our sample. From our spectra, it immediately turned out that HD 51110 is very He-weak (all He lines almost absent). We ascribe this chemical abundance anomaly to the gravitational settling of helium. This is only expected to show up in the stable, non-convective atmospheres of the higher gravity stars (white dwarfs and sub-dwarfs), as hypothesized by Michaud et al. (1983) and confirmed by Fabbian et al. (2005) for hot HB stars in NGC1904. The extreme strength of our observed $\mathrm{H} \gamma$ and $\mathrm{H} \alpha$ profiles are consistent with this hypothesis. Thus, we consider this star to be misclassified and hence unimportant for our study.

For HD 147670 (B8/B9 II) only H $\alpha$ was measured. Since we cannot derive stellar parameters from one line, we are forced to exclude it from our sample as well.

\section{B.2. Group I objects}

The first group of periodically variable B-type supergiants consists of those objects for which we were able to derive accurate results, on the basis of our assumptions, as discussed in Sects. 4.3 and 5. They are the following:

HD 168183 (O9.5 Ib) was already found to be a short-period (single-lined) binary, exhibiting large variations in radial velocity between consecutive nights (from -52 up to $92 \mathrm{~km} \mathrm{~s}^{-1}$ ) and having a period of about 4 days (Bosch et al. 1999). From our data we confirm both the short period and the huge radial velocity changes, going from $-60 \mathrm{~km} \mathrm{~s}^{-1}$ to $80 \mathrm{~km} \mathrm{~s}^{-1}$. The fact that HD 168183 is a member of the open cluster NGC 6611 allows us to derive its radius as $19 R_{\odot}$ (from $d=2.14 \pm 0.10-$ Belikov et al. 1999; $V=8.18, A_{V}=2.39-$ Hillenbrand et al. 1993).

Several different spectral types are mentioned for this star: B1 Ib/II (SIMBAD), B0 III (Evans et al. 2005), O9.5 I (Hillenbrand et al. 1993; confirmed by Bosch et al. 1999). We accept O9.5 I as the "true" spectral type, relying on our spectroscopically derived temperature of $30000 \mathrm{~K}$, which is in agreement with the observational $T_{\text {eff }}$ scale for O-type supergiants provided by Martins et al. (2005). From the $\mathrm{H} \alpha$ profile, we conclude that this object is of luminosity class Ib. The peculiar feature seen in the Si III 4574 line is a known instrumental defect, which is also present in the spectrum of HD 170938.

HD 89767 (B0 Ia). We obtain a reasonable fit for this object and no further comments are required.

HD 94909 (B0 Ia). This object was intensively discussed in the text (see Sect. 6).

HD 93619 (B0.5 Ib). From the fundamental parameters derived from the profile fits, this appears to be a typical B0.5 Ib star.
The small emission bump in the red wing of $\mathrm{H} \alpha$ seems to show up and vanish from one observation to the other.

HD 91943 (B0.7 Ib) For the discussion of this object, we refer to Sect. 6.

HD 96880 (B1 Ia). Also for this star the fit quality is good except for He I 6678, which would require a larger macroturbulence), and no further comments are required.

HD 115363 (B1 Ia). The observations of all targets discussed in this paper were done at the end of the lifetime of the CAT telescope. Unfortunately, at the time when it was completely closed down, in September 1998, we did not have Si measured for this target yet. He I 4471, which is a reasonable temperature estimator in the temperature range (at least if we adopt a solar helium content), gives us a perfect fit at a temperature of $20000 \mathrm{~K}$. Even more, HD 115363 is rather similar to HD 170938, which has the same spectral type and for which we do have Si III measured. For the latter object, we find exactly the same temperature and gravity. Taken together, we feel confident that the derived parameters are credible. Note also the perfect fit of the $\mathrm{H} \alpha$ emission.

HD 148688 (B1 Ia) has been fully discussed in the paper (see Sect. 6).

HD 170938 (B1 Ia). Although the emission peak observed in the $\mathrm{H} \alpha$ profile of HD 170938 is lower than the one observed in HD 115363, marking a difference in wind properties (with mass-loss rates differing within a factor of about two to four), all other stellar parameters are identical in both B1 Ia supergiants. Note that in this star we also detect the known instrumental defect in the Si III 4574 line.

HD 109867 (B1 Iab) is remarkable because of the very large changes in radial velocity, extending from -70 to $20 \mathrm{~km} \mathrm{~s}^{-1}$.

HD 154043 (B1 Ib). For this poorly known supergiant we are able to obtain a convincing fit. This is one of the few stars that does not show any radial velocity changes between the several line measurements. The radial velocity amounts to $-20 \mathrm{~km} \mathrm{~s}^{-1}$, and Si III appears to be asymmetric (see Sect. 4.1).

HD 106343 (B1.5 Ia). Apart from the fact that the blue part of $\mathrm{H} \alpha$ does not perfectly fit (both in the absorption trough and in the wings), we can be quite sure about the stellar parameters. The derived parameters confirm previous results from Lamers et al. (1995) and Bianchi et al. (1994) obtained by means of UV spectroscopy.

HD 111990 (B2 Ib). For this supergiant, part of a double system, only one $\mathrm{H} \alpha$ profile has been observed, which can be fitted very nicely. The P Cygni profile shows only a very small emission peak, which points to a moderate wind density. Note that from this star on we switch from the analysis of Si III to the analysis of Si II.

HD 92964 (B2.5 Iae). This object is one of the few sample stars that exhibits a clear asymmetry in the line profile, especially visible in $\mathrm{H} \gamma$. Since we are able to obtain an acceptable fit without further assumptions, we ascribe this asymmetric behaviour to the strong wind that is affecting the photospheric lines. As already mentioned, the He I 6678 line requires a 

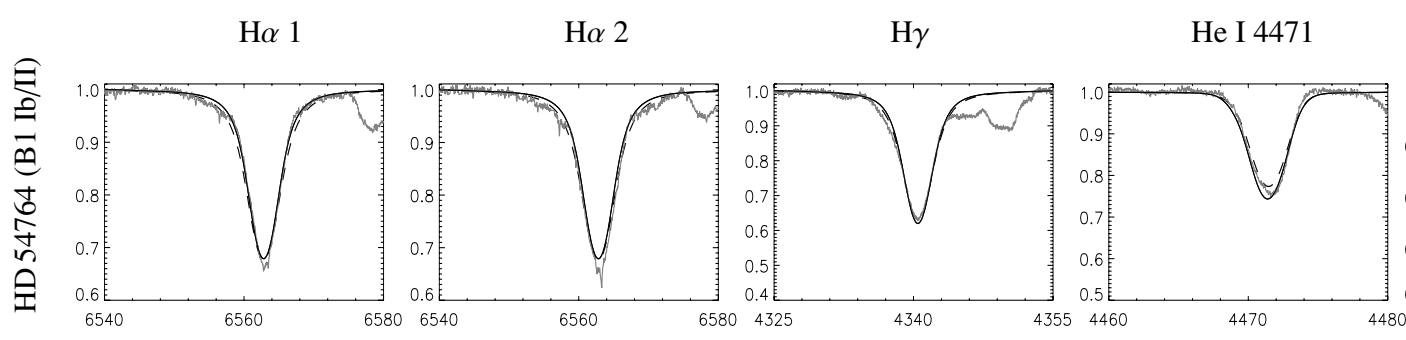

Si III 4552-4567-4574

Fig. B.1. Spectral line fits for HD 54764 - a prototype for supergiants belonging to group II: two models, located in completely different parameter domains (regarding $T_{\text {eff }}$ and $\log g$ ), produce similar line profiles. Bold: cool model $\left(T_{\text {eff }}=19000 \mathrm{~K}, \log g=2.4\right)$; dashed: hot model $\left(T_{\text {eff }}=\right.$ $26000 \mathrm{~K}, \log g=2.9)$.

macro-turbulent velocity twice as large as the one we derive from the Si lines.

HD 53138 (B3 Ia) has been discussed in the paper (see Sect. 6). Note that the fits and the parameters provided in Table 6 refer to the model at $17000 \mathrm{~K}$, which has been discussed as a compromise between the results obtained from our spectra and those obtained from the complete $J K T$ spectrum with additional ionisation stages from $\mathrm{Si}$ and $\mathrm{He}$.

HD 102997 (B5 Ia). The wind strength of the first measurement is lower than in the second observation, turning the P Cygni profile with a partly refilled absorption wing into a pure emission profile. Unfortunately we are unable to correctly reproduce this pure emission. This is the first object (out of two) for which we find an effective temperature larger than predicted from our calibration (see discussion at the end of Sect. 7).

HD 108659 (B5 Ib). Quite unknown among the supergiants, there is only one previous temperature estimate for this object, derived by Waelkens et al. (1998) using photometric measurements $\left(T_{\text {eff }}=11750 \mathrm{~K}\right)$. Our spectral fits indicate a much higher temperature, at least by $4000 \mathrm{~K}$. Although it seems that the derived mass-loss rate is too low (synthetic cores too deep), a further increase in $\dot{M}$ would result in a small red emission peak that is not observed. Also for this second B5 target, we find a temperature larger than expected for its spectral type (see Sect. 7).

HD 80558 (B6 Iab). As indicated especially by the second $\mathrm{H} \alpha$ profile, the wind of HD 80558 might show a non-spherical distribution, if one regards its shape as the beginning of a double-peaked structure. So far, the results of our wind analysis can be considered only as a very rough estimate.

HD 91024 (B7 Iab). From this spectral type on, He I 4471 becomes strongly sensitive to changes in effective temperature (see Fig. 4), at the expense of its reaction to changes in surface gravity. Thus, $T_{\text {eff }}$ is easily fixed at $12500 \mathrm{~K}$, with a gravity of $\log g=1.95$ following from the $\mathrm{H} \gamma$ line wings. Note that for this object the observed Si II components are of different strengths (as predicted), and thus could be fitted without any compromise.

HD 91024 has a moderate wind, which is slightly refilling the wings of $\mathrm{H} \alpha$. By inspection of the first $\mathrm{H} \alpha$ profile, we see an almost flat red wing (at continuum level), with a very steep decline into absorption. This also occurs for HD 106068, and cannot be represented by our synthetic profiles, at least at the inferred gravities.

HD 94367 (B9 Ia) has a moderately strong wind, displayed by the (strongly variable) emission peak of $\mathrm{H} \alpha$. Again, the overall shape of the profile cannot be modelled, and only the emission peak has been fitted.

\section{B.3. Group II objects}

We recall from Sect. 4.3 that, for some stars, the lack of Si II and/or Si IV prohibits us from choosing between two equally well fitting models at different positions in parameter space. To break this dichotomy, we proceed as follows. First of all, we aim at a good fit of He I 4471, which is a reasonable temperature indicator, though best suited only at the latest spectral types (below $15000 \mathrm{~K}$, see Fig. 4). Still, we have to keep in mind that this line depends on the (unknown) helium abundance. By combining the He I 4471 diagnostics (using solar helium abundances) with knowledge about the spectral type of the star, we are then able to obtain some clue about which temperature is the correct one.

HD 54764 (B1 Ib/II). We take HD 54764 as a prototypic example to illustrate the dichotomy problem in some detail. Relying on both the Si III triplet and the He I 4471 line, we are able to find two very different models that both give a reasonable match with the observations: $T_{\mathrm{eff}}=19000 \mathrm{~K}, \log g=2.45$ and $T_{\text {eff }}=26000 \mathrm{~K}, \log g=2.9$, respectively. By comparing the corresponding synthetic lines, one can hardly see any difference (see Fig. B.1), although the He I lines do favour the cooler solution.

Lyubimkov et al. (2004) derived values for $T_{\text {eff }}, \log g$, and $A_{V}$ for 102 stars, based on their colour indices. Although photometric methods rapidly lose their predictive capacity in the massive star domain, it is quite interesting to have a look at their results for HD 54764, which are $T_{\text {eff }}=25500 \pm 800 \mathrm{~K}$ and $\log g=3.56 \pm 0.17$ (note the rather optimistic error bars). Whereas this effective temperature would perfectly match our hotter model, the gravity is certainly way too high, which is immediately reflected in the Balmer line wings. Unfortunately we could not detect any He II or Si IV lines in the observed spectral range to unambiguously decide on $T_{\text {eff }}$. By concentrating on the secondary diagnostics mentioned above (spectral type), the cooler model is the more plausible one, if HD 54764 is actually of spectral type B1. Note that we find similar temperatures for all other B1 supergiants, also being in agreement with the former $T_{\text {eff }}$ calibration by Lennon et al. (1993). HD 54764 is one 

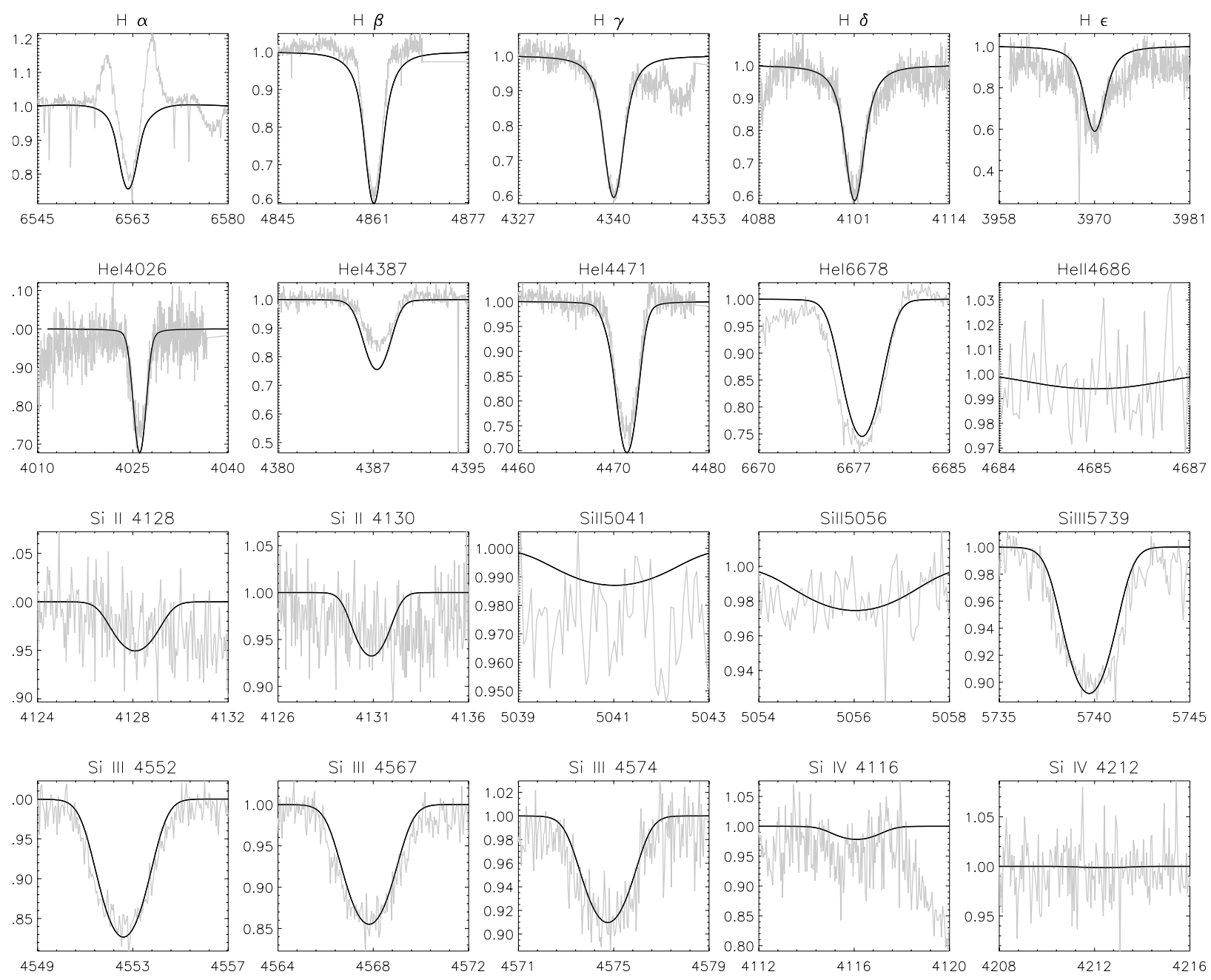

Fig. B.2. FEROS spectrum of HD 47240: synthetic line profiles at $T_{\text {eff }}=19000 \mathrm{~K}$ and $\log g=2.4$.

of the three supergiants within our sample that exhibits clear asymmetries in its line profile, in this case most likely because of an optical companion (Abt \& Cardona 1984).

HD 47240 (B1 Ib) is another example of finding two models with completely different stellar parameters, both fitting the Si III lines very well: one model with $T_{\text {eff }}=19000 \mathrm{~K}$ and $\log g=2.4$, and a second one with $T_{\text {eff }}=24000 \mathrm{~K}$ and $\log g=2.8$. Again, the He I lines are also too similar to allow for a clear distinction.

In contrast to the first group II object, for HD 47240 we do have a complete FEROS spectrum at our disposal, provided in GAUDI, the preparatory archive of ground-based observations in the framework of COROT (Solano et al. 2005). The drawback of this spectrum is its very low $\mathrm{S} / \mathrm{N}$, so that the $\mathrm{Si}$ II and Si IV lines, although being very different in strength, still lie in the noise level. At least from He II 4686, on the other hand, the hotter solution can be ruled out with high confidence, since in this case the line is predicted to be much stronger than it is observed. Again, also for this object, its spectral type points towards the cooler solution. The complete comparison with the FEROS observations is given in Fig. B.2. It is worth mentioning that this star might be a binary and that the $\mathrm{H} \alpha$ morphology (double-peaked structure) is typical for a fast rotator observed almost equator-on (Morel et al. 2004). Note that we do observe this structure also for the fast rotators HD 64760 and HD 157246.

HD 141318 (B2 II). For this object, the differences between the parameters of the cool and the hot solutions are not as large as for the previous cases, namely $\left(T_{\text {eff }}, \log g\right)=(20000 \mathrm{~K}, 2.9)$ and ( $22000 \mathrm{~K}, 3.2)$, respectively. Although the forbidden component of He I 4471 is fitting slightly better for the hotter model, the $\mathrm{Si}$ III triplet is better represented for the cooler one. Note that an intermediate model at $21000 \mathrm{~K}$ does not give a good fit. From its spectral type then, we prefer the cooler solution.

\section{B.4. Group III objects}

Group III is constituted of those (three) stars for which we cannot claim a similar accuracy as obtained for the previous two groups, either because they are somewhat extreme, or because of their peculiar spectrum that complicates a reliable fit, or a combination of both. All three objects belong to the group of 10 stars that were added to the sample of Waelkens et al. (1998), and are possible chemically peculiar stars. We have classified these stars as "unreliable", and the derived parameters have to be considered with caution.

HD 105056 (B0 Iabpe) is a nitrogen-rich, carbon-deficient supergiant, exhibiting very strong emission lines, up to twice 
the continuum level, and has been classified as an ON9.7 Iae supergiant in several studies. Its peculiar nature and the extremely dense wind hamper a correct modelling of the photospheric lines (e.g., $\mathrm{H} \gamma$ ), since these are severely contaminated by the wind. Although nitrogen enrichment usually goes along with the enrichment of helium, we used a compromise solution for He I 4471 and 6678 at normal abundance, guided by the temperature we found from fitting the Si III triplet.

HD 98410 (B2.5 Ib/II). Similar to the last object, also HD 98410 is an extreme supergiant, with a very strong wind, $\mathrm{H} \alpha$ completely in emission, and strongly refilled photospheric lines. Because of the restricted wavelength range around each line, the normalisation of the $\mathrm{H} \alpha$ profile became more problematic than usual, thus increasing the uncertainty of the derived mass-loss rate. The difficulty to fit $\mathrm{H} \alpha$ and $\mathrm{H} \gamma$ in parallel might point to the presence of strong clumping in the wind (e.g., Repolust et al. 2004), and the actual mass-loss rate might be considerably lower than implied by $\mathrm{H} \alpha$. Taken together with the peculiar shape of one of these profiles (which might be explained by an equatorially enhanced wind), we "classify" our analysis as unreliable.

HD 68161 (B8 Ib/II?). Although the spectroscopically derived spectral type is B8 Ib/II, Eggen (1986) mentioned that photometry indicates a different luminosity class, namely B8 IV. HD 68161 has been considered by Paunzen \& Maitzen (1998) as a probable, variable chemically peculiar star, in particular a variable star of the $\alpha^{2} \mathrm{CVn}$ type. If so, this star should be a main sequence star of spectral type later than B8p (consistent with the photometrically derived spectral type), exhibiting a strong magnetic field and abnormally strong lines of Si among other elements. Since for this star we could not observe the Si lines, we cannot confirm this conjecture. Note, however, that the derived gravity is not so different from HD 46769, which is a "normal" B8 Ib supergiant, and that it is also in agreement with typical gravities for these objects. Due to the discussed uncertainties we add this object to our list of unreliable cases.

\section{B.5. Group IV objects - the comparison stars}

Group IV is the group of 12 bright comparison stars, selected from the Bright Star Catalogue, previously not known to exhibit any periodic variability. For these objects three lines have mostly been observed: He I 4471, $\mathrm{H} \gamma$, and $\mathrm{H} \alpha$, which, in combination with our $T_{\text {eff }}$ calibration for B-supergiants (see Sect. 7 and Table 5), will be used to estimate the required stellar and wind parameters.

HD 149038 (09.7 Iab). While SIMBAD lists this star as a B0 supergiant, a spectral type of O9.7 Iab has been suggested by Walborn \& Bohlin (1996), Lamers et al. (1999), Maíz-Apellániz et al. (2004), and Fullerton et al. (2006). Recent revisions of stellar parameter calibrations in the O-type regime (Martins et al. 2005 ) predict $T_{\mathrm{eff}} \approx 30500 \mathrm{~K}, \log g \approx 3.2, R \approx 22.1 R_{\odot}$, and $\log L / L_{\odot} \approx 5.57$ for an 09.5 supergiant, where these values have been used by Fullerton et al. (2006). The latter authors additionally revised the distance of this star, from $1.3 \mathrm{kpc}$ (Georgelin et al. 1996) to $1.0 \mathrm{kpc}$. The radio mass-loss rate, $\log \dot{M}_{\text {radio }}$ (at time of observation), could be constrained as being less than $-5.51 \pm 0.18 M_{\odot} / \mathrm{yr}$, with a terminal velocity of $v_{\infty}=1750 \mathrm{~km} \mathrm{~s}^{-1}$ and $\beta=1.0$. Our calibration predicts somewhat lower effective temperatures at 09.7 , but the general agreement between our values and those stated above is convincing.

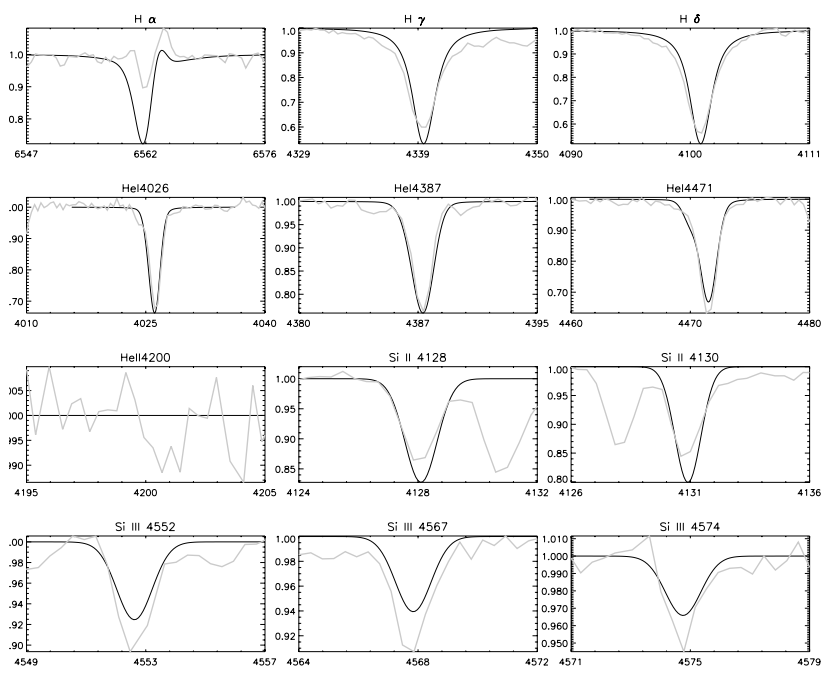

Fig. B.3. Spectral line fits for the $J K T$ spectrum of HD 58350. Gray: observed $J K T$ spectrum (Lennon et al. 1993), black: predictions at $T_{\text {eff }}=$ $13500 \mathrm{~K}$ and $\log g=1.75$.

HD 64760 (B0.5 Ib) is a very fast rotating supergiant. With a projected rotational velocity, $v \sin i$, of more than $220 \mathrm{~km} \mathrm{~s}^{-1}$, it is rather likely being observed at a very high inclination, i.e., almost equator-on. Due to its particularly interesting wind structure, HD 64760 is amongst the best studied early B-type supergiants (Prinja et al. 2002; Kaufer et al. 2002, 2006, being the most recent investigations). Its richness in spectral features led to conclusive evidence for the existence of a corotating two and four armed spiral structure, suggested to be originating from stream collisions at the surface and perturbations in the photoshere of the star (Kaufer et al. 2002, 2006). H $\alpha$ consists of a double-peaked structure, with a blueward and a somewhat stronger, redward shifted emission peak around the central absorption (as in Fig. 2 of Kaufer et al. 2002). This star is clearly a Ib supergiant, with a moderate mass-loss rate refilling the photospheric absorption. Of course, we are not able to reproduce this double-peaked profile, and only the gravity can be derived (together with some crude estimate for $\dot{M}$ ) for the adopted effective temperature, $T_{\text {eff }}=24000 \mathrm{~K}$, which is $\log g=3.2$. From the $M_{V}$ calibration, we finally have $R_{*}=24 R_{\odot}$, where all these parameters are similar to those as reported by Howarth et al. (1998).

HD 157246 (B1 Ib). This is another example of a rotationally modulated wind, similar to HD 64760. We find a projected rotational velocity of $275 \mathrm{~km} \mathrm{~s}^{-1}$. The $\mathrm{H} \alpha$ profile shows the typical blue- and redward shifted emission peaks, which are about equal in height. They suggest that the wind is equatorially compressed, see also Prinja et al. (1997). With only two lines at our disposal (i.e., $\mathrm{H} \alpha$ and $\mathrm{H} \gamma$ ), we can only suggest that the stellar parameters are consistent with those reported until now (Prinja et al. 2002).

HD 157038 (B1/2 IaN) is enriched in nitrogen and helium $(n(\mathrm{He}) / n(\mathrm{H}) \approx 0.2$ according to our analysis), and the observed lines can be fitted reasonably well with a model of $T_{\text {eff }}=20000 \mathrm{~K}$ and $\log g=2.3$.

HD 165024 (B2 Ib). As for HD 157246, we have only two hydrogen lines at our disposal, and we can derive only $\log g$ and an estimate of the wind properties. As we have no direct 
means to derive the rotational velocity, we can only give a range for $v_{\text {macro, }}$, depending on the adopted $v \sin i$ from the literature. The most reasonable solution is a combination of $v \sin i=120 \mathrm{~km} \mathrm{~s}^{-1}$ and $v_{\text {macro }}=50 \mathrm{~km} \mathrm{~s}^{-1}$ (considering the macrotubulence derived for objects of similar spectral type).

HD 75149 (B3 Ia) is a poorly studied early B supergiant, with a dense wind. All lines can be fitted at an effective temperature of $16000 \mathrm{~K}$.

HD 58350 (B5 Ia). HD 58350 or $\eta \mathrm{CMa}$ is a well-studied B-type supergiant. By means of the NLTE atmosphere code TLUSTY (Hubeny \& Lanz 2000), McErlean et al. (1999) have also analysed this star (cf. Sect. 6.2). Their best-fitting model has a temperature of $16000 \mathrm{~K}$, whereas previous temperature estimates were lower, between 13000 and 14000 K. From our calibrations (Table 5), we find a typical value of $13500 \mathrm{~K}$ at B5, which is just consistent with these lower values and also gives an acceptable fit. On the other hand, the $J K T$ spectrum (from the online LDF atlas) shows that the effective temperature might be actually higher by $1000 \mathrm{~K}$ (still within the quoted error bars), since Si II is too strong and Si III too weak (see Fig. B.3).

HD 86440 (B5 Ib). No comment necessary, except that the forbidden component of He I 4471 also fits very well, at an effective temperature of $13500 \mathrm{~K}$.
HD 125288 (B6 Ib). This is one other object for which we find a perfect fit of the forbidden He I 4471 component.

HD 106068 (B8 Iab). This rarely studied bright B supergiant shows exactly the same feature in $\mathrm{H} \alpha$ as we have found for HD 91024 (group I): a very flat red wing, with a sudden steep decrease into the (blue-shifted) absorption. Of course, we cannot fit such a profile.

HD 46769 (B8 Ib). For B8 objects, our calibration gives an effective temperature of $12100 \mathrm{~K}$, which is also required to fit the He I 4471 line (remember its sensitivity to $T_{\text {eff }}$ in this temperature domain). Models with a temperature increased by $1000 \mathrm{~K}$, compared to our calibration, would give a too strong He I 4471 line, and vice versa for lower temperatures.

HD 111904 (B9 Ia) is another poorly known object from the BSC. Contrary to HD 106068, the flat wing is in the blue part of the line this time and not on the red side, i.e., there is some (unknown) refilling mechanism. The star, being a member of the open star cluster NGC 4755 in Crux, allows us to derive an $M_{V}$ of $-7.38 \pm 0.15$ (Slowik \& Peterson 1995) (consistent with our calibration within the adoted errors), and hence a radius of $95 R_{\odot}$. 